\title{
THE FIRST SPECTRUM OF XENON
}

\author{
By William F. Meggers, T. L. deBruin, and C. J. Humphreys
}

\section{ABSTRACT}

Although 30 years have passed since the discovery of xenon, comparatively little has been known about its spectra, and all attempts to analyze the structure of the $\mathrm{Xe}_{1}$ spectrum have been foiled by the incompleteness and inaccuracy of the available data. This situation has been improved, as follows:

Discharge tubes were filled with pure Xe gas and operated so as to emit only the spectrum of neutral xenon atoms. This spectrum was photographed with quartz-prism and with diffraction grating spectrographs from the ultra-violet to the infra-red. Wave lengths ranging from $3,442.77$ to $10,107.2 \mathrm{~A}$ have been determined for 318 lines and are supplemented by estimates of relative intensity. Analysis of these new data has resulted in the identification of spectral terms, combinations of which account for practically all of the observed lines.

The normal state of the neutral Xe atom $\left(s^{2} p^{6}\right)$ is represented by a single level, and successive excited states arise from the addition of $s-, p-, d-, f$ - electrons to the xenon ion $\left(s^{2} p^{5}\right)$. In the notation first introduced to describe the $\mathrm{Ne}_{\mathrm{I}}$ spectrum, and subsequently used for $\mathrm{A}_{1}, \mathrm{Kr}_{1}$, and now also for $\mathrm{Xe}_{1}$, the single level is called " $p_{0}$ ", and the term groups produced by addition of $s-, p-, d-, f$ - electrons are designated " $s$," " $p$," " $d$ and $s$," " $x$ and $y$," respectively. These groups in the order named theoretically consist of $4,10,12$, and 12 levels. Each group divides into two subgroups converging in the limit to the two levels of a ${ }^{2} \mathrm{P}$ term characterizing the normal state of the xenon ion. This subdivision is striking in $\mathrm{Kr}_{1}$, but especially so in $\mathrm{Xe}_{\mathrm{I}}$ on account of the large separation $(9,621)$ of the $\mathrm{Xe}_{\mathrm{II}_{1}}$ doublet.

From the limits of the most regular series in the $\mathrm{Xe}_{\mathrm{I}}$ spectrum, and from certain combinations, the absolute value of the $1 s_{5}$ term is established as $30,766.98$, and all other term values are based on this one. The resonance lines in the extreme ultra-violet $(1,469.61$ and $1,295.56 \mathrm{~A})$ then fix the value of the largest term $p_{0}=$ 97,835 from which the ionization potential of 12.078 volts is deduced for neutral xenon atoms.

\section{CONTENTS}

I. Introduction

732

II. Wave-length measurements

III. Analysis of the $\mathrm{Xe}_{\mathrm{I}}$ spectrum

1. Theoretical terms

2. Absolute term values; ionization potential of $\mathrm{Xe}_{-}$

3. The principal series $\left(1 s_{\mathrm{i}}=m p_{\mathrm{k}}\right)$

4. The "fundamental" series $(X, Y, Z, U, V$, and $W)$

5. The subordinate series

6. The $\mathrm{Xe}_{\mathrm{I}}$ spectrum in the extreme ultra-violet

7. Identification of the experimental terms

8. List of $\mathrm{Xe}_{\mathrm{x}}$ lines. 


\section{INTRODUCTION}

Xenon, the heaviest and rarest of the atmospheric gases, was discovered in 1898 by Ramsay and Travers ${ }^{1}$ who announced that:

This gas possesses a spectrum analogous in character to that of argon, but differing entirely in the position of the lines. With the ordinary discharge the gas shows three lines in the red and about five very brilliant lines in the blue; while with the jar and spark gap these lines disappear and are replaced by four brilliant lines in the green, intermediate in position between the two groups of argon lines, the glow in the tube changing from blue to green.

This first reference to the spectrum of xenon points out that there are two entirely different spectra depending on the conditions of excitation, and it has since been the custom to refer to that excited without capacity and external spark gap as the first spectrum, while that produced by a disruptive discharge is called the second spectrum. A prismatic spectrogram showing 15 lines of the second spectrum of xenon was reproduced in color by Ramsay and Travers ${ }^{2}$ in 1901. Approximate wave lengths of 250 lines (3,454 to 6,596 A) were published in 1901 by Liveing and Dewar, ${ }^{3}$ but this list includes lines of both spectra. The most extensive and accurate descriptions of xenon spectra were published in 1903 by Baly, ${ }^{4}$ who measured 92 lines $(2,536.58$ to $6,198.70 \mathrm{~A})$ in the first spectrum and 1,247 lines $(2,414.88$ to $6,097.80 \mathrm{~A})$ in the second, a small number of lines being common to both. Eleven of Baly's lines for the first spectrum are identifiable with mercury, so that 81 lines remain in his list $(3,469.95$ to $6,198.70 \mathrm{~A}$ ). No extension to Baly's list was made until 1919 , when Merrill ${ }^{5}$ reported the wave lengths of 54 new lines in the red and infra-red $(5,931.2$ to $9,162.76 \mathrm{~A})$. Ten strong lines of the first spectrum $(4,500.978$ to $4,923.246 \mathrm{~A})$ and two of the second $(4,603.028$ and $4,844.333 \mathrm{~A}$ ) were measured relative to the primary standard $(6,438.4696 \mathrm{~A}$ of $\mathrm{Cd})$ by Meggers, ${ }^{6}$ and three of the former (4,624.275, $4,671.225$, and $4,734.154 \mathrm{~A}$ ) were also investigated and confirmed by Perard. ${ }^{7}$

Forty-two xenon lines $(4,078$ to 6,768 A) were examined with Lummer-Gehrcke interferometer plates by Gehrcke and Janicki; ${ }^{8}$ they reported fine structure for only two lines, namely, 4,501.13 and 4,734.30 A.

The resonance lines of xenon $(1,469.61$ and 1,295.56 A) were discovered in 1926 by Hertz and Abbink, ${ }^{9}$ and a more extensive investigation of $\mathrm{Xe}$ spectra in the extreme ultra-violet was subsequently

\footnotetext{
1 Ramsay and Travers, Rep. British Assoc., p. 828; 1898

${ }^{2}$ Ramsay and Travers, Phil. Trans, A 197, p. 47; 1901.

${ }^{3}$ Liveing and Dewar, Proc. Roy. Soc., 68, p. 389; 1901.

' Baly, Phil. Trans., A 202, p. 183; 1903.

- Merrill, B. S. Sci. Paper, 15, p. 251 (No. 345); 1919.

- Meggers, B. S. Sci. Paper, 17, p. 193 (No. 414); 1921.

7 Perard, Comptes rendus, 184, p. 681; 1927.

8 Gehrcke and Janicki, Ann. d. Phys., 81, p. 314; 1926.

- Hertz and Abbink, Natuurwiss., 14, p. 648; 1926.
} 
made by Abbink and Dorgelo, ${ }^{10}$ who published the wave lengths for 15 lines $(1,469.61$ to $1,027.04 \mathrm{~A}$ ) assigned to the first spectrum of $\mathrm{Xe}$.

The electrodeless discharge for xenon was investigated by $\mathrm{L}$. Bloch, E. Bloch, and Déjardin ${ }^{11}$ for the purpose of separating successive stages of ionization. Among 1,000 lines, $91(3,550$ to $7,120 \mathrm{~A})$ were assigned to the arc spectrum or first spectrum of xenon (from neutral atoms), and the remaining lines were ascribed to singly, doubly, or trebly ionized atoms. This method of excitation appears to favor the production of spark lines, but it is, in general, a very inefficient source of arc lines. The Zeeman effect of Xe lines has not been investigated at all.

Many attempts have been made to find regularities in the first spectrum of xenon, but on account of inadequate data most of these have failed to yield results of any value. In 1914 Paulson ${ }^{12}$ published four triplets, two of which are now known to involve true terms. Sommer ${ }^{13}$ arranged 142 lines (including mercury impurity lines) in pairs and groups with 25 wave number differences ranging from 58 to $5,840 \pm 3$. It appears that a large majority of these "constant" differences are fortuitous and have no physical significance. Hicks ${ }^{14}$ and Williams ${ }^{15}$ have also discussed the structure of the first spectrum of xenon, but their results, too, are mostly spurious. Finally, McLennan and Ruedy ${ }^{16}$ made absorption experiments with excited xenon gas and found two infra-red lines (8,232 and 8,819 A) to show absorption. Assuming that these lines involved the metastable level $s_{5}$, they succeeded in establishing a number of $s p$ combinations, but the data available did not permit the assignment of any line to a definite transition.

The above-mentioned attempts to analyze the structure of the $\mathrm{Xe}_{\mathrm{I}}$ spectrum emphasize the importance of having a satisfactory description of the spectrum, and our efforts have been directed toward this end in connection with similar work on the $\mathrm{Kr}_{I}$ spectrum. ${ }^{17}$

The purest krypton obtainable contained a trace of xenon, and it was therefore considered advisable to study the xenon spectra also. Furthermore, the practical reason for our study of krypton spectra originates with a proposal to adopt a $\mathrm{Kr}$ line as a primary standard of wave length ${ }^{18}$ instead of the $\mathrm{Cd}$ line now recognized as such. The argument that $\mathrm{Kr}$ lines are sharper than $\mathrm{Cd}$ lines applies with even greater force to $\mathrm{Xe}$ lines with which it is theoretically possible at low temperatures to produce interference phenomena with retarda-

\footnotetext{
${ }^{10}$ Abbink and Dorgelo, Zeit, f. Phys., 47, p. 221; 1928.

11 L. Bloch, E. Bloch, Dejardin, Ann. de. Phys. (10), 2, p. 461; 1924.

12 Paulson, Astrophys, J., 40, p. 307; 1914.

13 Sommer, Zeit. f. Phys., 13, p. 85; 1923.

14 Hicks, Phil. Trans., A 220, p. 335; 1919.

15 Williams, Ciencias Fisicas y Matematicas (No. 82), p. 255; 1928.

16 McLennan and Ruedy, Trans. Roy. Soc. Can. (3), 22, p. 15; 1928.

17 Meggers, deBruin and Humphreys, B. S. Jour. Research, 3 (No. 89), p. 129; 1929,

88 Conf. Gen. Poids et Mesures, p. 52; 1927.
} 
tions of a meter. The possibilities in this direction appear to be worth investigating, and the present paper may be regarded as a contribution to such a program. It is obviously desirable in the first place to describe the first spectrum of xenon with sufficient completeness and accuracy to permit analysis of its structure and classification of its lines. Certain properties of spectral lines are now known to be related to spectral terms (atomic energy levels), so that a classification of the lines enables one to draw upon theoretical considerations in a discussion of their suitability as standards of wave length. The present paper, therefore, deals with a preliminary description and with the interpretation of the $\mathrm{Xe}_{\mathrm{I}}$ spectrum. It presents new values for the wave lengths and estimated intensities of 318 lines characterizing neutral xenon atoms and identifies spectral terms which, by their combinations, account for practically all of the observed lines. From the limits of term sequences and from combinations the absolute values of all terms are established. The largest term is ${ }^{1} S_{0}\left(p_{0}\right)$ representing the normal state of the neutral xenon atom; its value is 97,835 , corresponding to an ionization potential of 12.078 volts. The structure of this spectrum is compared with that of the analogous spectrum of the preceding rare gases, $\mathrm{Ne}, \mathrm{A}, \mathrm{Kr}$, and a certain grouping of the terms is seen to be associated with the coordination of terms to limits representing the ground levels of the ions. This analysis of the $\mathrm{Xe}_{\mathrm{I}}$ spectrum has uncovered additional evidence that $f$-type electrons (in addition to $s-, p$-, and $d$-electrons) may play a part in the production of rare gas spect,tral terms.

It will be desirable to supplement this description and analysis with investigations to record fainter lines and far infra-red lines, to compare the wave lengths of the stronger lines with the primary standard or with the meter itself, to study the hyperfine structure of complex lines, and to observe the Zeeman effects.

\section{WAVE-LENGTH MEASUREMENTS}

The sources of radiation, spectrographs, and procedure employed in this study of the $\mathrm{Xe}_{\mathrm{I}}$ spectrum were almost identical with those described in our report on the $\mathrm{Kr}_{\mathrm{I}}$ spectrum, so that it will suffice to give a brief summary of them here. Glass discharge tubes with aluminum disk electrodes like those described by Nutting ${ }^{19}$ were filled with remarkably pure Xenon gas purchased from the Linde Gesellschaft für Eismaschinen in Höllriegelskreuth b. München. These tubes were operated on a. c. transformers rated to give 10,000 volts in one case and 40,000 volts in the other. The current in the tube in either case was about 20 milliamperes. The pressure of gas in the tubes and condition of operation was such that an almost 


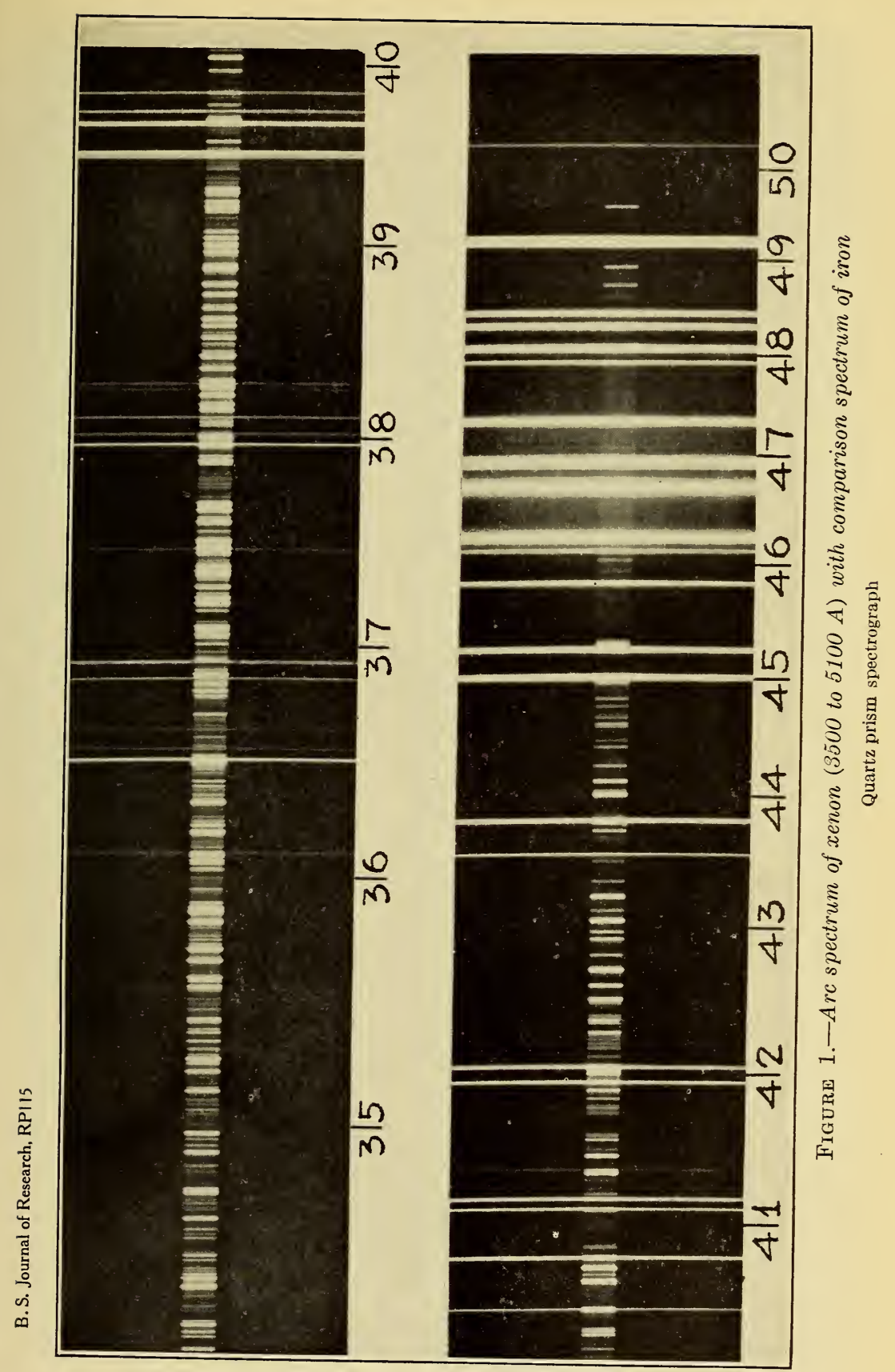




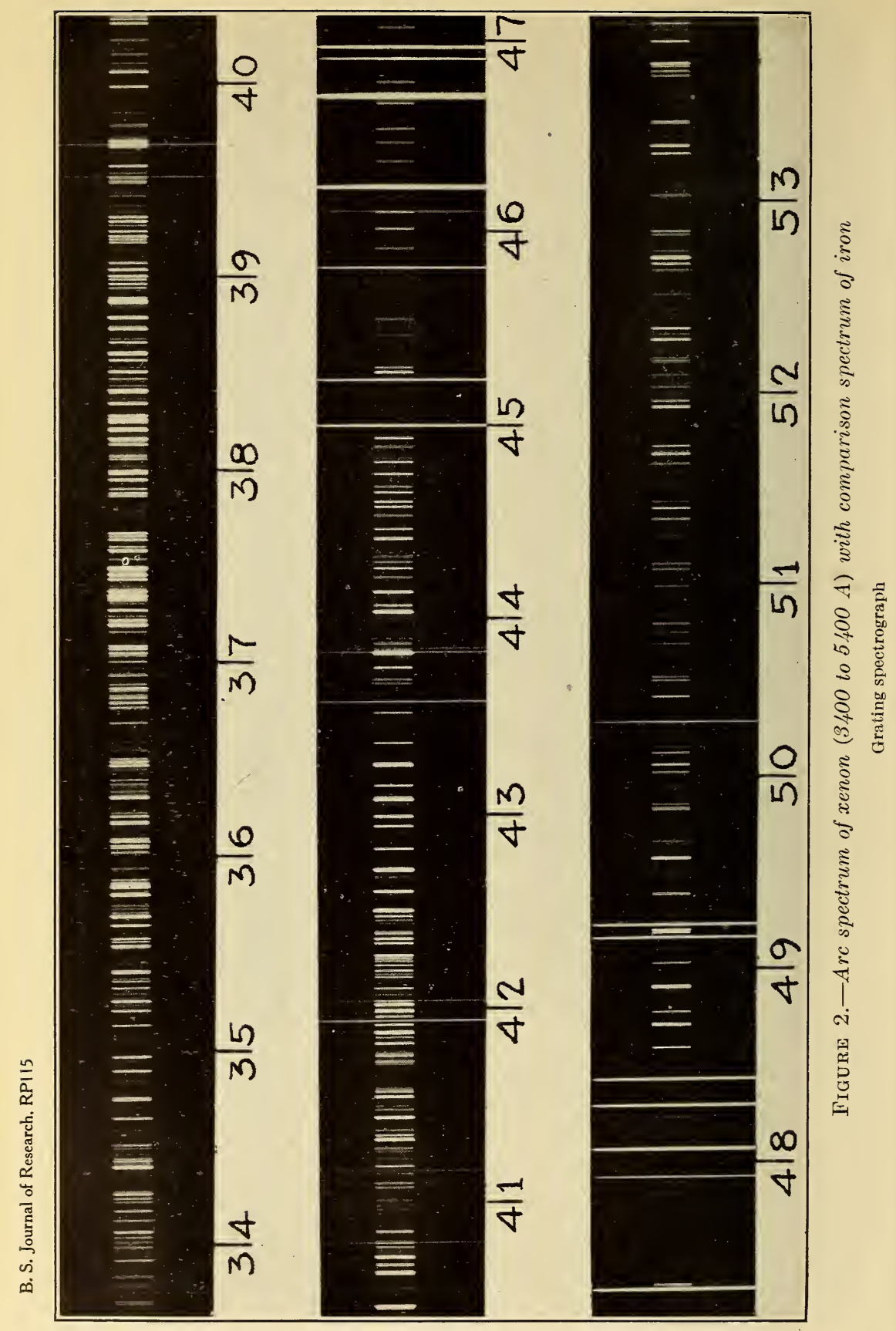




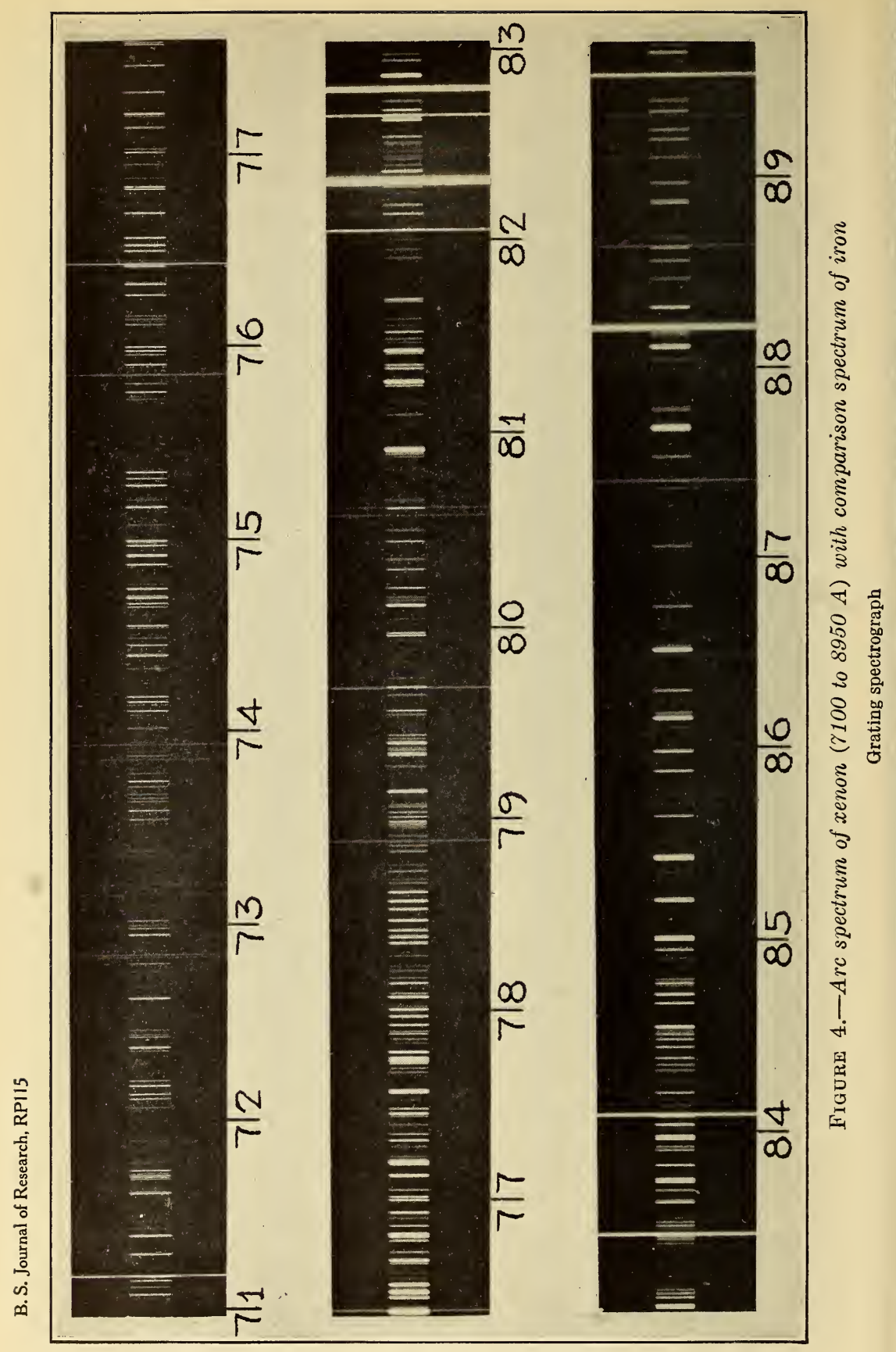




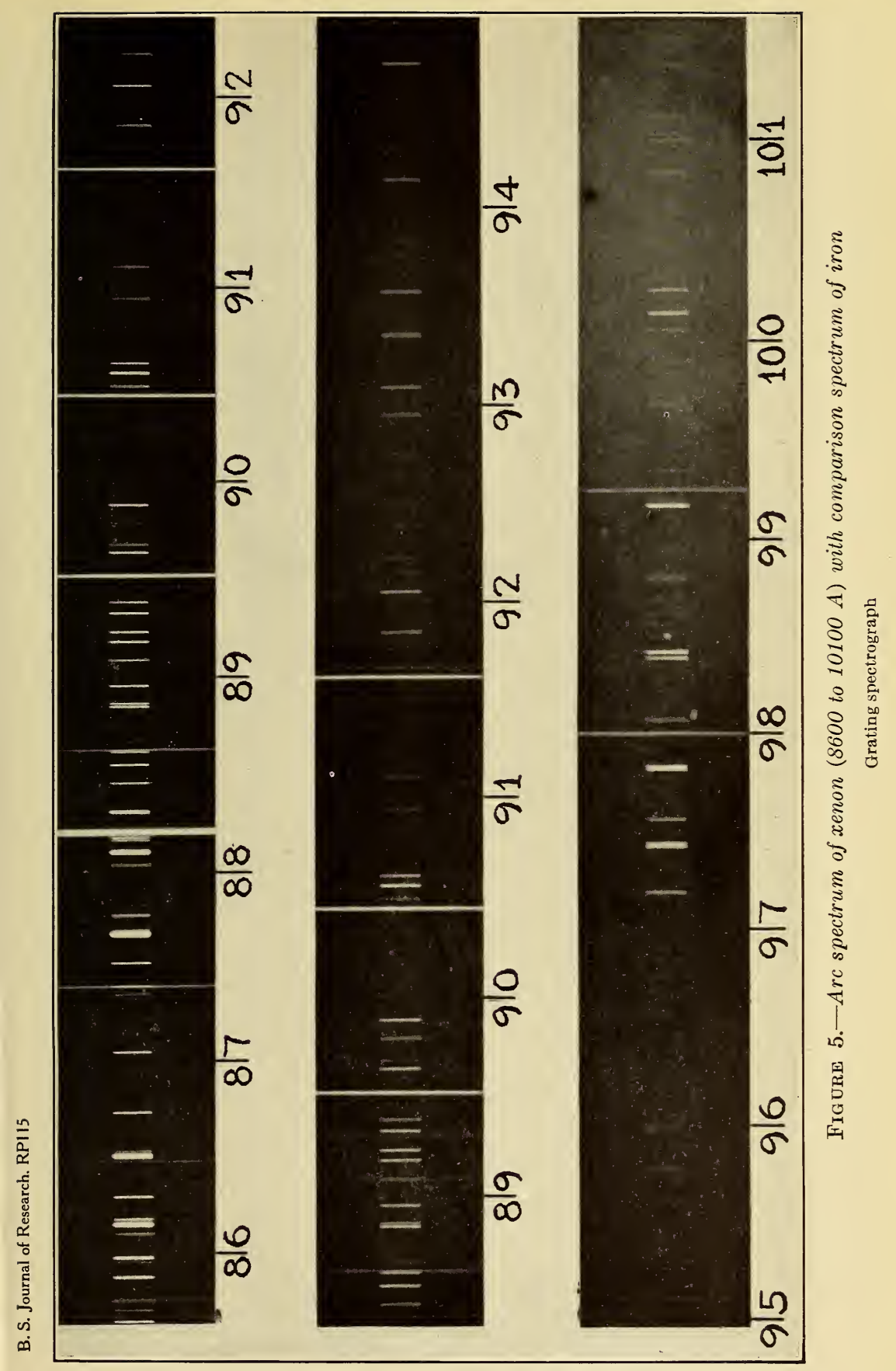


perfectly pure arc spectrum $\left(\mathrm{Xe}_{\mathrm{I}}\right)$ was emitted, only traces of three or four most intense spark lines $\left(\mathrm{X}_{\mathrm{II}}\right)$ showing on the longest exposures. Electrode evaporation occurs when the discharge is passing so that the bulbs are opaque after 10 to 20 hours, but the tubes are good sources of $\mathrm{Xe}$ spectra for several hundred hours operation. Most of the spectrograms were made in the first and second order of a 6-inch grating of 21 feet radius of curvature, mounted stigmatically as described before. ${ }^{20}$ These were supplemented by several made with a Hilger $E_{1}$ autocollimating quartz spectrograph to record faint lines in the ultra-violet end of the spectrum. Eastman's No. 33 photographic plates were used regularly except for the longer wave regions, where No. 36 plates bathed in photosensitizing solutions of dyes were employed. The exposure times ranged from 10 to 48 hours, the latter exposure being sufficient to record a line in the infra-red at $10107.2 \mathrm{~A}$.

Comparison spectra of iron were impressed upon the plates before and after each of the long exposures to xenon and wave-length measurements ${ }^{21}$ were made relative to the secondary standards in the spectrum of the iron arc. Most of the lines were measured on four or five spectrograms, and the average probable error of the mean in such cases is not more than one or two hundredths of an Angstrom unit. Some faint lines were observed only once; that is, on the strongest exposure. The wave lengths of such lines are not given beyond one decimal place. The observed wave lengths, estimated intensities, and term combinations of the $\mathrm{Xe}_{\mathbf{I}}$ spectrum are presented in Table 14 at the end of this paper.

Since no complete photographic map of the xenon arc spectrum has ever been published, we are reproducing portions of our spectrograms in Figures 1, 2, 3, 4, and 5. These show the first spectrum of xenon from its limit in the ultra-violet near $3400 \mathrm{~A}$ to the infra-red near $10000 \mathrm{~A}$. Xe resembles the preceding rare gases, $\mathrm{Ne}, \mathrm{A}$, and $\mathrm{Kr}$, in that its first spectrum consists of groups of very intense lines, one in the blue (4501 to $4923 \mathrm{~A}$ ) and another beginning at $8206 \mathrm{~A}$ in the infra-red and probably. extending far beyond the photographic limit. The intervening region is well filled with lines of medium and lower intensity, the strongest visible lines being 5824, 6182, and $6470 \mathrm{~A}$. When the tables of wave length were first compiled, it was rather surprising to find that about 50 per cent more lines were observed for the $\mathrm{Xe}_{\mathrm{I}}$ spectrum than for the $\mathrm{Kr}_{\mathrm{I}}$ spectrum which was produced and studied in essentially the same manner. The explanation of this superior abundance of $\mathrm{Xe}_{\mathrm{I}}$ lines is found in the analysis of the $\mathrm{Xe}_{\mathrm{I}}$ spectrum. (See the fundamental series.) 


\section{ANALYSIS OF THE Xe $\mathrm{I}_{\mathrm{I}}$ SPECTRUM}

\section{THEORETICAL TERMS}

Neutral xenon $(Z=54)$ has in the unexcited state, the atomic configuration of a closed shell with two $5 s$ - and six $5 p$ - electrons, symbolized by $5 s^{2} 5 p^{6}$. The spectral term which characterizes the normal state is a single level, ${ }^{1} S_{0}$ according to Hund's theory. ${ }^{22}$ The xenon ion, $\mathrm{Xe}^{+}$, is characterized by the electron group $s^{2} p^{5}$, giving rise to an inverted doublet term ${ }^{2} \mathrm{P}_{2,1}{ }^{23}$ in the $\mathrm{Xe}_{\mathrm{II}}$ spectrum, and the terms representing the excited states of the neutral atom arise from the interaction of the series electron with the $s^{2} p^{5}$ group. The theoretical terms which may be expected to account for the first spectrum of xenon are thus obtained by adding in turn the electrons $n s, n p, n d$, and $n f$ to the term ${ }^{2} \mathrm{P}$, as shown in Table 1 .

$\mathrm{T}_{\mathrm{ABLE}}$ 1.-Electron configurations and theoretical terms of the $X e_{\mathrm{I}}$ spectrum

\begin{tabular}{|c|c|c|c|c|c|}
\hline $\begin{array}{l}\text { Electron con- } \\
\text { figuration }\end{array}$ & \multicolumn{4}{|c|}{ Theoretical terms based on ${ }^{2} \mathrm{P}_{2,1}$ of $\mathrm{Xe}_{\mathrm{II}}$} & $\begin{array}{c}\text { Number of } \\
\text { levels }\end{array}$ \\
\hline $\begin{array}{lll}s^{2} & p^{6} & \\
\left(s^{2}\right. & \left.p^{5}\right) & s \\
\left(s^{2}\right. & \left.p^{5}\right) & p \\
\left(s^{2}\right. & \left.p^{5}\right) & d \\
\left(s^{2}\right. & \left.p^{5}\right) & f\end{array}$ & $\begin{array}{ll}{ }^{1} \mathrm{~S}_{0} & \\
{ }^{1} \mathrm{P}_{1} & \\
{ }^{1} \mathrm{~S}_{0}{ }^{1} \mathrm{P}_{1} & \\
& 1 \\
{ }^{1} \mathrm{D}_{2} \\
{ }^{1} \mathrm{P}_{1}{ }^{1} \mathrm{D}_{2}{ }^{1} \mathrm{~F}_{3} \\
{ }^{1} \mathrm{D}_{2}{ }^{1} \mathrm{~F}_{3}{ }^{1} \mathrm{G}_{4}\end{array}$ & $\begin{array}{l}{ }^{3} \mathrm{~S}_{2} \\
{ }^{3} \mathrm{P}_{2,1,0} \\
{ }^{3} \mathrm{D}_{3,2,1}\end{array}$ & $\begin{array}{l}{ }^{3} \mathrm{P}_{2,1,0} \\
{ }^{3} \mathrm{P}_{2,1,0} \\
{ }^{3} \mathrm{D}_{3,2,1} \\
{ }^{3} \mathrm{~F}_{4,3,2}\end{array}$ & $\begin{array}{l}{ }^{3} \mathrm{D}_{3,2,1} \\
{ }^{3} \mathrm{~F}_{4,3,2} \\
{ }^{3} \mathrm{G}_{5,4,3}\end{array}$ & $\begin{array}{r}1 \\
4 \\
10 \\
12 \\
12\end{array}$ \\
\hline
\end{tabular}

Paschen ${ }^{24}$ in his analysis of the $\mathrm{Ne}_{\mathrm{I}}$ spectrum, introduced a special notation for these levels. The set of four levels resulting from the addition of an $s$-electron were called " $s$-terms," $s_{2}, s_{3}, s_{4}, s_{5}$ in the order of increasing magnitude. The set of 10 levels was referred to as

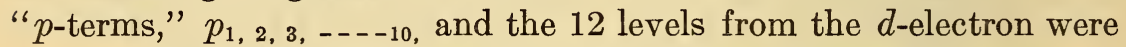
represented in part as " $d$-terms" and the remainder as "s-terms." Two terms of the following group (originating with an $f$-electron) were recognized and designated as "X- and Y-terms." The same notation was used by Meissner ${ }^{25}$ in his analysis of the $A_{1}$ spectrum and by the authors ${ }^{26}$ in their description of the $\mathrm{Kr}_{\mathrm{I}}$ spectrum. On account of certain difficulties and uncertainties in the translation of this Paschen notation into the modern quantum symbols, we shall retain the older notation also for the discussion of the $\mathrm{Xe}_{\mathrm{r}}$ spectrum. This facilitates intercomparison of these four spectra but it is important to remember that homologous terms in the different spectra may have different inner quantum numbers. In all cases $\mathrm{Ne}_{\mathrm{I}}, \mathrm{A}_{\mathrm{I}}, \mathrm{Kr}_{\mathrm{I}}$, and $\mathrm{Xe}_{\mathrm{I}}$, the $4 s$-terms are strictly comparable, but if the $10 p$-terms

\footnotetext{
22 Hund, Linienspektren und periodisches System der Elemente, p. 144, Julius Springer, Berlin; 1927.

${ }^{23}$ For convenience in printing and reading the inner quantum numbers of levels belonging to even multiplicities are increased by one-half.

${ }^{24}$ Paschen, Ann. d. Phys., 60, p. 405; 1919; 63, p. 201; 1920.

28 Meissner, Zeit. f. Phys., 27, p. 238; 1926; 39, p. 172; 1926; 40, p. 839; 1927

28 Meggers, de Bruin and Humphreys, B. S. Jour. Research, 3 (No. 89), p. 129; 1929.
} 
are numbered (with subscripts) in order of magnitude they must be rearranged to compare levels with the same inner quantum numbers as shown in Table 2.

TABLE 2.-Corresponding terms in $N e_{\mathrm{I}}, A_{\mathrm{I}}, K r_{\mathrm{I}}$, and $X e_{\mathrm{I}}$ spectra

\begin{tabular}{|c|c|c|c|c|c|c|c|c|c|c|c|c|c|c|}
\hline $\mathbf{j}$ & 0 & 1 & 0 & 2 & 1 & 2 & 1 & 2 & 3 & 1 & 1 & 0 & 1 & 2 \\
\hline $\mathrm{NeI}$ & $p_{1}$ & $p_{2}$ & $p_{3}$ & $p_{4}$ & $p_{5}$ & $p_{6}$ & $p_{7}$ & $p_{8}$ & $p_{9}$ & $p_{10}$ & $s_{2}$ & $s_{3}$ & $s_{4}$ & $s_{5}$ \\
\hline $\mathrm{AI}$ & $p_{1}$ & $p_{2}$ & $p_{5}$ & $p_{3}$ & $p_{4}$ & $p_{6}$ & $p_{7}$ & $p_{8}$ & $p_{9}$ & $p_{10}$ & $s_{2}$ & $s_{3}$ & $s_{4}$ & $s_{5}$ \\
\hline $\mathrm{KrI}$ & $p_{1}$ & $p_{3}$ & $p_{5}$ & $p_{2}$ & $p_{4}$ & $p_{6}$ & $p_{7}$ & $p_{8}$ & $p_{9}$ & $p_{10}$ & $s_{2}$ & $s_{3}$ & $s_{4}$ & $s_{5}$ \\
\hline $\mathrm{XeI}$ & $p_{1}$ & $p_{2}$ & $p_{5}$ & $p_{3}$ & $p_{4}$ & $p_{6}$ & $p_{7}$ & $p_{9}$ & $p_{8}$ & $p_{10}$ & $s_{2}$ & $s_{3}$ & $s_{4}$ & $s_{5}$ \\
\hline
\end{tabular}

In each case it has been found that the group of terms may be divided into subgroups converging to two limits separated by a "displacement constant," $A$. The limits are the two ground levels, ${ }^{2} P_{2}$ and ${ }^{2} P_{1}$ of the ion. The displacement constant, $A$, found from the limits of the Ritzian and non-Ritzian series, is related to the coordination of the arc spectrum levels to the two levels ${ }^{2} P_{2,1}$ of the ion and is approximately the same magnitude as the separation of these levels. The data for the four rare gas spectra are given in Table 3.

TABLE 3.-Displacement constants and level separations in rare gas spectra

\begin{tabular}{|c|c|c|c|c|}
\hline & Neon & Argon & Krypton & Xenon \\
\hline${ }_{2}^{A} P_{2,1}$ & $\begin{array}{l}780.0 \\
782\end{array}$ & $\begin{array}{l}1,423.20 \\
1,431\end{array}$ & $\begin{array}{l}5,330 \pm \\
5,371\end{array}$ & $\begin{array}{l}\text { Vide infra. } \\
9,621\end{array}$ \\
\hline 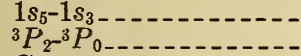 & 776. 90 & $1,409.92$ & $5,219.90$ & $9,129.24$ \\
\hline $\begin{array}{l}\text { Centers of gravity of } \\
3 p \text {-term groups }\end{array}$ & 788 & 1,451 & 5,353 & Vide infra. \\
\hline
\end{tabular}

On account of the very large separation of ${ }^{2} P_{2,1}$ for xenon, it is impossible to find any but the first members of the non-Ritzian sequence $n p_{1,2,3,4}$, since the second members would give negative term values. It is therefore not possible to determine the displacement constant $A$ of xenon from the series limits. However, an upper limit can be found from the distance between centers of gravity of the ${ }^{2} p_{5,6} \ldots--10$ and the ${ }^{2} p_{1,2,3,4}$ groups. This upper limit has the value 10,540 . The lower limit for this $A$ is the $1 s_{5}-1 s_{3}$ separation, which amounts to $9,129.24$. The ${ }^{2} P_{2,1}$ separation in $\mathrm{Xe}_{\mathrm{II}}$ has been found ${ }^{27}$ to be 9,621 , which is in satisfactory agreement with the two limits 9,129 and 10,540 just discussed. For calculating the effective quantum numbers of the non-Ritzian $\mathrm{X}_{\mathrm{I}}$ terms we have assumed $\mathrm{A}=9,621$.

${ }^{27}$ Humphreys and deBruin, Sci., 68, p. 573; 1928. 


\section{ABSOLUTE TERM VALUES; IONIZATION POTENTIAL OF Xe}

Among the various series identified in $\mathrm{Ne}_{\mathrm{I}}, \mathrm{A}_{\mathrm{I}}$, and $\mathrm{Kr}_{\mathrm{I}}$ spectra, one, namely, $2 p_{9}-m d_{4}{ }^{\prime}$, was unique in the regularity of its effective quantum numbers. The analogous series in $\mathrm{Xe}_{\mathrm{I}}$ is $2 p_{8}-m d_{4}^{\prime}$, and again it is the most regular as well as the longest. As in the preceding cases, this series has been employed for the determination of absolute term values, since it is probable that the best values must result from extrapolation of the longest series following most closely a Ritz formula. The $2 p_{8}-m d_{4}{ }^{\prime}$ series fixes the level $2 p_{8}=19,431.49$, and addition of the wave number $1 s_{5}-2 p_{8}=11,335.49$, places the $1 s_{5}$ level at $30,766.98$. The same level can be arrived at independently from several different series. The results are summarized as follows:

1. Series $2 p_{8}-m d_{4}^{\prime}$ and $1 s_{5}-2 p_{8}$

$$
1 s_{5}=30,766.98
$$

2. Series $2 p_{9}-m d_{4}$ and $1 s_{5}-2 p_{9}$

3. Series $2 p_{6}-m d_{1}{ }^{\prime}$ and $1 s_{5}-2 p_{6}$

$$
1 s_{5}=30,768.26
$$

4. Series $1 s_{3}-m X$
and $1 s_{5}-1 s_{3}$

4. Series $1 s_{3}-m X$
and $1 s_{5}-1 s_{3}$

$$
1 s_{5}=30,767.61
$$

$$
1 s_{5}=30,766.12
$$

5. Series $1 s_{5}-m p_{6}$

$$
1 s_{5}=30,767.24
$$

6. Series $1 s_{5}-m p_{8}$

$$
1 s_{5}=30,765.28
$$

The mean of the six values is $30,766.91$, practically identical with the first one, which is chosen as the basic value. All remaining term values are relative to $1 s_{5}=30,766.98$, and any error in this level is therefore the same for all the other terms. The details of the abovementioned calculations of $1 s_{5}$ are presented in Table 4. 
$\mathrm{T}_{\mathrm{ABLE}}$ 4.-Determination of absolute term values

Series $2 p_{8}-m d_{4}^{\prime}$

$$
\begin{aligned}
\nu=A-\frac{R}{[m+\mu+a(A-\nu)]^{2}} \\
R_{x e}=109,736.66 \\
A=19431.489 \\
\alpha=-0.000001377271113 \\
\mu=+0.5197276575 \pm 10^{-10}
\end{aligned}
$$

\begin{tabular}{|c|c|c|c|c|c|}
\hline$m$ & Int. & $\lambda_{\text {obs. }}$ & Dobs. & $\nu_{\text {calce }}$ & $\begin{array}{l}\nu \text { obs. }- \\
\nu \text { calc. }\end{array}$ \\
\hline $\begin{array}{l}2 \\
3 \\
4 \\
5\end{array}$ & $\begin{array}{r}3 \\
30 \\
30\end{array}$ & $\begin{array}{l}9,513.33 \\
7,119.58 \\
6,318.08\end{array}$ & $\begin{array}{l}10,508.71 \\
14,041.91 \\
15,823.22\end{array}$ & $\begin{array}{l}a 1,809.645 \\
10,511.369 \\
14,041.910 \\
15,823.220\end{array}$ & $\begin{array}{r}-2.66 \\
0.00 \\
0.00\end{array}$ \\
\hline $\begin{array}{l}6 \\
7 \\
8 \\
9\end{array}$ & $\begin{array}{l}8 \\
3 \\
1 \\
1-\end{array}$ & $\begin{array}{l}5,934.14 \\
5,716.14 \\
5,579.16 \\
5,486.90\end{array}$ & $\begin{array}{l}16,846.98 \\
17,489.48 \\
17,918.88 \\
18,220.18\end{array}$ & $\begin{array}{l}16,847.045 \\
17,489.456 \\
17,918.929 \\
18,220.180\end{array}$ & $\begin{array}{r}-0.06 \\
+0.02 \\
-0.05 \\
0.00\end{array}$ \\
\hline
\end{tabular}

\begin{tabular}{|c|c|c|c|c|c|}
\hline$m$ & Int. & $\lambda_{\text {obs. }}$ & $\nu_{\text {obs. }}$ & $\nu_{\text {calc. }}$ & $\begin{array}{l}\nu \text { obs. }- \\
\nu \text { calc. }\end{array}$ \\
\hline $\begin{array}{l}2 \\
3 \\
4 \\
5\end{array}$ & $\begin{array}{r}5 \\
60 \\
20\end{array}$ & $\begin{array}{l}9,167.47 \\
6,882.15 \\
6,182.41\end{array}$ & $\begin{array}{l}10,905.14 \\
14,526.35 \\
16,170.45\end{array}$ & $\begin{array}{r}4,036.67 \\
11,233.42 \\
14,472.71 \\
16,170.45\end{array}$ & $\begin{array}{r}-328.28 \\
+53.64 \\
0.00\end{array}$ \\
\hline $\begin{array}{l}6 \\
7 \\
8 \\
9\end{array}$ & $\begin{array}{l}4 \\
2 \\
1- \\
1-\end{array}$ & $\begin{array}{l}5,824.76 \\
5,618.80 \\
5,488.56 \\
5,400.4\end{array}$ & $\begin{array}{l}17,163.33 \\
17,792.47 \\
18,214.69 \\
18,512.01\end{array}$ & $\begin{array}{l}17,163.33 \\
17,792.08 \\
18,214.69 \\
18,512.15\end{array}$ & $\begin{array}{r}0.00 \\
+0.39 \\
0.00 \\
-0.14\end{array}$ \\
\hline
\end{tabular}

$$
\begin{aligned}
2 p_{8} & =19,431.489 \\
1 s_{5}-2 p_{8} & =11,335.49 \\
1 s_{5} & =30,766.98
\end{aligned}
$$

Series $2 p_{9}-m d_{4}$

$$
\begin{aligned}
& A=19,716.015 \\
& \alpha=+0.000006775882325 \\
& \mu=+0.5392837788 \pm 10^{-10}
\end{aligned}
$$

$$
\begin{aligned}
2 p_{9} & =19,716.015 \\
1 s_{5}-2 p_{9} & =\frac{11,052.25}{1 s_{5}}=30,768.26
\end{aligned}
$$

a Exact calculation given on p. 748 . 
TABLE 4.-Determination of absolute term values-Continued

Series $2 p_{6}-m d_{1}{ }^{\prime}$

$$
\begin{aligned}
& A=18,622.663 \\
& \alpha=+0.000002752635528 \\
& \mu=+0.6180082686 \pm 10^{-10}
\end{aligned}
$$

\begin{tabular}{|c|c|c|c|c|c|}
\hline$m$ & Int. & $\lambda_{\text {obg. }}$ & $\nu_{\text {obs }}$ & $\nu_{\text {eale. }}$ & $\begin{array}{l}\nu \text { obs. }- \\
\nu \text { calc. }\end{array}$ \\
\hline $\begin{array}{l}2 \\
3 \\
4 \\
5\end{array}$ & $\begin{array}{r}2 \\
6 \\
20\end{array}$ & $\begin{array}{l}9,685.29 \\
7,393.77 \\
6,595.54\end{array}$ & $\begin{array}{l}10,322.11 \\
13,521.19 \\
15,157.85\end{array}$ & $\begin{array}{r}3,121.40 \\
10,344.02 \\
13,508.22 \\
15,157.85\end{array}$ & $\begin{array}{r}-21.91 \\
+12.97 \\
0.00\end{array}$ \\
\hline $\begin{array}{l}6 \\
7 \\
8 \\
9\end{array}$ & $\begin{array}{l}4 \\
2 \\
1 \\
1-\end{array}$ & $\begin{array}{l}6,200.86 \\
5,974.08 \\
5,830.47 \\
5,733.4\end{array}$ & $\begin{array}{l}16,122.35 \\
16,734.35 \\
17,146.52 \\
17,436.83\end{array}$ & $\begin{array}{l}16,122.35 \\
16,734.34 \\
17,146.52 \\
17,437.20\end{array}$ & $\begin{array}{r}0.00 \\
+0.01 \\
0.00 \\
-0.37\end{array}$ \\
\hline
\end{tabular}

$$
\begin{aligned}
2 p_{6} & =18,622.663 \\
1 s_{5}-2 p_{6} & =\frac{12,144.95}{1 s_{5}}=\overline{30,767.61}
\end{aligned}
$$

\begin{tabular}{|c|c|c|c|c|c|}
\hline$m$ & Int. & $\lambda_{\text {obs }}$ & 2 obs. & $\nu_{\text {cale }}$ & $\begin{array}{l}\nu \text { obs. - } \\
\nu \text { calc. }\end{array}$ \\
\hline $\begin{array}{l}3 \\
4 \\
5 \\
6 \\
7\end{array}$ & $\begin{array}{r}40 \\
15 \\
4 \\
2 \\
1-\end{array}$ & $\begin{array}{l}6,827.30 \\
5,823.89 \\
4,392.74 \\
5,162.66 \\
5,023.80\end{array}$ & $\begin{array}{l}14,643.04 \\
17,165.90 \\
18,538.30 \\
19,364.48 \\
19,899.71\end{array}$ & $\begin{array}{l}14,639.10 \\
17,165.90 \\
18,538.30 \\
19,364.48 \\
19,899.71\end{array}$ & $\begin{array}{r}+3.94 \\
0.00 \\
0.00 \\
0.00 \\
0.00\end{array}$ \\
\hline
\end{tabular}

Series $1 s_{3}-m X$

$A=21,636.880$

$\alpha=+0.000002293149515$

$\mu=+0.9439590749 \pm 10^{-10}$

$$
\begin{aligned}
1 s_{3} & =21,636.880 \\
1 s_{5}-1 s_{3} & =9,129.24 \\
\hline 1 s_{5} & =30,766.12
\end{aligned}
$$

\begin{tabular}{|c|c|c|c|c|c|}
\hline$m$ & Int. & $\lambda_{\mathrm{ob} q}$ & $\nu_{\text {obs. }}$ & $\nu_{\text {calc }}$ & $\begin{array}{l}\nu \text { obs. - } \\
\nu \text { calc. }\end{array}$ \\
\hline 2 & 300 & $8,231.62$ & $12,144.95$ & $12,353.55$ & -208.60 \\
\hline 3 & 150 & $4,624.29$ & $21,618.90$ & $21,654.54$ & -35.64 \\
\hline 4 & 15 & $3,950.92$ & $25,303.42$ & $25,303.42$ & 0.00 \\
\hline 5 & 3 & $3,685.91$ & $27,122.65$ & $27,122.65$ & 0.00 \\
\hline 6 & 1 & $3,549.84$ & $28,162.27$ & $28,162.27$ & 0.00 \\
\hline 7 & $1-$ & $3,469.96$ & $28,810.56$ & $28,812.44$ & -1.88 \\
\hline
\end{tabular}

Series $1 s_{5}-m p_{6}$

$A=30,767.237$

$\alpha=-0.000003114322468$

$\mu=+0.4985618368 \pm 10^{-10}$

$1 s_{5}=30,767.24$ 
TABLE 4.-Determination of absolute term values-Continued

Series $1 s_{5}-m p_{8}$

$A=30,765.284$

$\alpha=-0.000004234844672$

$\mu=+0.4630334435 \pm 10^{-10}$

\begin{tabular}{|r|r|r|r|r|r|}
\hline$m$ & Int. & \multicolumn{1}{|c|}{$\lambda_{\text {obs. }}$} & \multicolumn{1}{|c|}{$\nu_{\text {obs. }}$} & \multicolumn{1}{|c|}{$\nu_{\text {oalo. }}$} & \multicolumn{1}{c|}{$\begin{array}{c}\nu \text { obs. } \\
\nu \text { calc. }\end{array}$} \\
\hline & & & & & \\
2 & 200 & $8,819.43$ & $11,335.49$ & $11,409.57$ & -74.08 \\
3 & 300 & $4,671.22$ & $21,401.71$ & $21,401.71$ & 0.00 \\
4 & 20 & $3,967.55$ & $25,197.37$ & $25,197.37$ & 0.00 \\
5 & 3 & $3,963.51$ & $27,066.84$ & $27,067.19$ & -0.35 \\
6 & 1 & $3,554.03$ & $28,129.07$ & $28,129.07$ & 0.00 \\
7 & $1-$ & $3,472.49$ & $28,790.06$ & $28,790.61$ & -0.55 \\
\hline
\end{tabular}

$1 s_{5}=30,765.28$

The resonance lines of xenon lie in the extreme ultra-violet at 1469.61 and $1295.56 \mathrm{~A}$. They form the connecting link between the ground term $p_{0}\left({ }^{1} \mathrm{~S}_{0}\right)$ and the excited states $1 s_{2}$ and $1 s_{4}\left({ }^{1} \mathrm{P}_{1}\right.$ and $\left.{ }^{3} \mathrm{P}_{1}\right)$, and permit the evaluation of this largest term, and calculation of the ionization potential. Thus, from the terms $1 s_{4}=29,789.34$ and $1 s_{2}=20,649.44$, we obtain:

$$
\begin{aligned}
& p_{0}-1 s_{4}=1469.61 \mathrm{~A} ; \nu=68,045.3 ; p_{0}=97,834.6 \\
& p_{0}-1 s_{2}=1295.56 \mathrm{~A} ; \nu=77,186.7 ; p_{0}=97,836.1
\end{aligned}
$$

The final value of the normal state of neutral xenon atoms is therefore

$$
p_{0}\left({ }^{1} \mathrm{~S}_{0}\right)=97,835.3
$$

corresponding to an ionization potential of 12.078 volts. This is in good agreement with the estimation of Abbink and Dorgelo ${ }^{28}$ on the absorption of spark lines (ca. 12.0 volts) but somewhat higher than the value obtained by Hertz and Kloppers ${ }^{29}$ from critical potential measurements (ca. 11.5 volts).

\section{THE PRINCIPAL SERIES $1 s_{\mathrm{i}}-m p_{\mathrm{k}}$}

The data for the 10 principal series $1 s_{1}-m p_{\mathrm{k}}$ are presented in Table 5, where the observational data are represented by estimated intensity of the spectral line (in parenthesis) followed by the measured wave length $\lambda$. The corresponding wave number in vacuum, $\nu$, was computed with the aid of Kayser's "Tabelle der Schwingungszahlen," except for lines in the unexplored infra-red region which are represented by term differences. Values of $m p$ have been calculated from each combination, and finally the mean value of each term and the effective quantum number $\sqrt{\frac{R}{m p}}$, associated with it are given. 
TABLE 5.-Xe principal series, $1 s_{\mathrm{i}}-m_{\mathbf{k}}$

Series, $m p_{1}$

Limits: $1 s_{2}=20,649.44$

$1 s_{4}=29,789.34$

\begin{tabular}{|c|c|c|}
\hline Comb. & $m$ & 2 \\
\hline$s_{2} p_{1}$ & $\left\{\begin{array}{c}\lambda \\
\nu \\
m p_{1}\end{array}\right.$ & $\begin{array}{r}(8) 7,887.38 \\
12,675.00 \\
7,974.44\end{array}$ \\
\hline $\begin{array}{l}s_{4} p_{1} \\
\text { Mean }\end{array}$ & $\left\{\begin{array}{c}\lambda \\
\nu \\
m p_{1} \\
m p_{1}\end{array}\right.$ & $\begin{array}{r}(30) 4,582.74 \\
21,814.92 \\
7,974.42 \\
7,974.43\end{array}$ \\
\hline & $\sqrt{\frac{i}{m p_{1}+A}}$ & 2. 4973 \\
\hline
\end{tabular}

$A=9,621$

Series, $m p_{2}$

Limits: $1 s_{2}=20,649.44$

$1 s_{3}=21,637.74$

- $1 s_{4}=29,789.34$

$1 s_{5}=30,766.98$

\begin{tabular}{|c|c|c|}
\hline Comb. & $m$ & 2 \\
\hline$s_{2} p_{2}$ & $\begin{array}{c}\lambda \\
\nu \\
m p_{2}\end{array}$ & $\begin{array}{r}(20) 8,266.53 \\
12,093.65 \\
8,555.79\end{array}$ \\
\hline$s_{3} p_{2}$ & $\begin{array}{c}\lambda \\
\nu \\
m p_{2}\end{array}$ & $\begin{array}{r}(15) 7,642.02 \\
13,081.95 \\
8,555.79\end{array}$ \\
\hline$s_{4} p_{2}$ & $\begin{array}{c}\lambda \\
\nu \\
m p_{2}\end{array}$ & $\begin{array}{r}\text { (2) } 4,708.19 \\
21,233.66 \\
8,555.68\end{array}$ \\
\hline$s_{5} p_{2}$ & $\begin{array}{c}\lambda \\
\nu \\
m p_{2}\end{array}$ & $\begin{array}{r}(100) 4,500.99 \\
22,211.12 \\
8,555.86\end{array}$ \\
\hline Mean & $\begin{array}{c}m p_{2} \\
\sqrt{\frac{R}{m p_{2}+A}}\end{array}$ & $\begin{array}{r}8,555.78 \\
2.4571\end{array}$ \\
\hline
\end{tabular}

$A=9,621$ 
TABLE 5.-Xe principal series, $1 s_{\mathrm{i}}-m_{\mathrm{k}}-$ Continued

Series, $m p_{3}$

$$
\text { Limits: } \begin{aligned}
& 1 s_{2}=20,649.44 \\
& 1 s_{4}=29,789.34 \\
& 1 s_{5}=30,766.98
\end{aligned}
$$

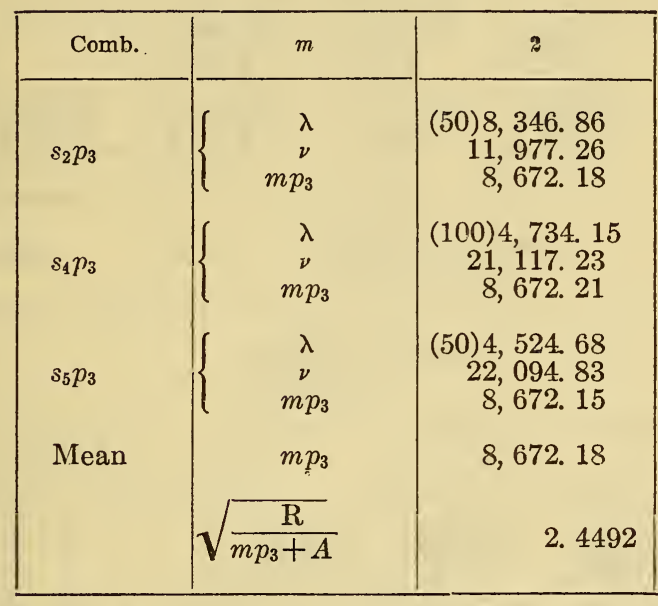

$$
A=9,621
$$

Series, $m p_{4}$

$$
\text { Limits: } \begin{aligned}
1 s_{2}=20,649.44 \\
1 s_{3}=21,637.74 \\
1 s_{4}=29,789.34 \\
1 s_{5}=30,766.98
\end{aligned}
$$

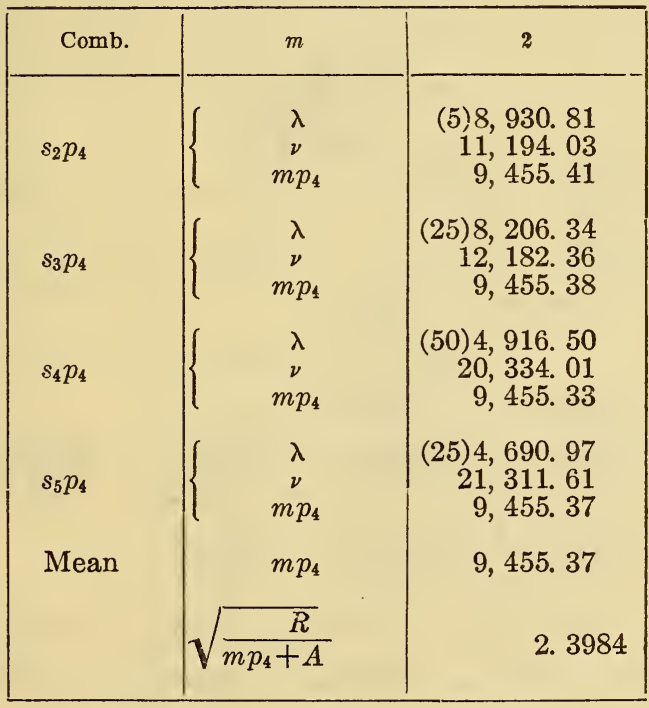

$$
A=9,621
$$

Note.-The use of 6-point type for the remaining series does not reflect on their importance. 


\section{$\mathrm{T}_{\triangle B \mathrm{BLE}}$ 5.-Xe principal series, $1 s_{\mathrm{i}}-m_{\mathrm{k}}-$ Continued}

\begin{tabular}{|c|c|c|c|c|c|c|}
\hline \multicolumn{7}{|c|}{$\begin{aligned} & \text { Series, } m p_{5} \\
& \text { Limits: } 1 s_{2}=20,649.44 \\
& 1 s_{4}=29,789.34\end{aligned}$} \\
\hline Comb. & $m$ & 2 & 3 & 4 & 5 & 6 \\
\hline$s_{2} p_{5}$ & $\begin{array}{c}\lambda \\
\nu \\
m p_{5}\end{array}$ & $\begin{array}{r}2,933.85 \\
17,715.59\end{array}$ & $\begin{array}{r}(5) 8,576.03 \\
11,657.21 \\
8,992.23\end{array}$ & $\begin{array}{r}(15 d) 6,504.16 \\
15,370.54 \\
5,278.90\end{array}$ & & \\
\hline$s_{4} p_{5}$ & $\stackrel{\lambda}{\lambda}$ & $\begin{array}{r}(150) 8,280.16 \\
12,073.75 \\
17,715.59\end{array}$ & $\begin{array}{r}(100) 4,807.01 \\
20,797.16 \\
8,992.18\end{array}$ & $\begin{array}{r}(20) 4,078.81 \\
24,510.06 \\
5,279.28\end{array}$ & $\begin{array}{r}(4) 3,809.90 \\
26,239.99 \\
3,549.35\end{array}$ & $\begin{array}{r}(2) 3,669.94 \\
27,240.67 \\
2,548.67\end{array}$ \\
\hline Mean & $m p_{5}$ & $17,715.59$ & $8,992.20$ & $5,279.09$ & $3,549.35$ & $2,548.67$ \\
\hline & $\sqrt{\frac{R}{m p_{3}}}$ & 2.4888 & 3. 4933 & 4. 5593 & 5. 5604 & 6.5618 \\
\hline
\end{tabular}

Series, $m p_{6}$

Limits: $1 s_{2}=20,649.44$

$1 s_{4}=29,789.34$

$1 s_{s}=30,766.98$

\begin{tabular}{|c|c|c|c|c|c|c|c|}
\hline Comb. & $m$ & 2 & 3 & 4 & 5 & 6 & 7 \\
\hline$s_{2} p_{6}$ & $\begin{array}{c}\lambda \\
m p_{6}\end{array}$ & $\begin{array}{r}2,027.40 \\
18,622.04\end{array}$ & $\begin{array}{r}(3) 8,692.08 \\
11,501.57 \\
9,147.87\end{array}$ & $\begin{array}{r}(3) 6,583.15 \\
15,186.11 \\
5,463.33\end{array}$ & & & \\
\hline$s_{4} p_{6}$ & $\begin{array}{c}\lambda \\
\nu \\
m p_{0}\end{array}$ & $\begin{array}{r}(50) 8,952.25 \\
11,167.31 \\
18,622.03\end{array}$ & $\begin{array}{r}(60) 4,843.29 \\
20,641.37 \\
9,147.97\end{array}$ & $\begin{array}{r}(10) 4,109.71 \\
24,325.78 \\
5,463.56\end{array}$ & $\begin{array}{r}(2) 3,823.76 \\
26,144.88 \\
3,644.46\end{array}$ & $\begin{array}{r}(4) 3,677.62 \\
27,183.79 \\
2,605.55\end{array}$ & \\
\hline$s_{5} p_{6}$ & 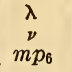 & $\begin{array}{r}(300) 8,231.64 \\
12,144.92 \\
18,622.06\end{array}$ & $\begin{array}{r}(200) 4,624.29 \\
21,618.90 \\
9,148.06\end{array}$ & $\begin{array}{r}(20) 3,950.92 \\
25,303.43 \\
5,463.55\end{array}$ & $\begin{array}{r}(4) 3,685.91 \\
27,122.65 \\
3,644.33\end{array}$ & $\begin{array}{r}(2) 3,549.84 \\
28,162.27 \\
2,604.71\end{array}$ & $\begin{array}{r}\text { (1) } 3,469.96 \\
28,810.55 \\
1,956.43\end{array}$ \\
\hline Mean & $m p_{6}$ & $18,622.04$ & $9,147.97$ & $5,463.48$ & $3,644.40$ & $2,604.93$ & $1,956.43$ \\
\hline & $\sqrt{\frac{R}{m p_{6}}}$ & 2.4275 & 3. 4635 & 4. 4817 & 5.4874 & 6.4905 & 7.4893 \\
\hline
\end{tabular}

Series, $m p_{7}$

Limits: $1 s_{2}=20,649.44$

$1 s_{3}=21,637.74$.

$1 s_{4}=29,789.34$

$1 s_{5}=30,766.98$

\begin{tabular}{|c|c|c|c|c|c|c|}
\hline Comb. & $m$ & 2 & 3 & 4 & 5 & 6 \\
\hline$s_{2} p_{7}$ & $\begin{array}{c}\lambda \\
\nu \\
m p_{7}\end{array}$ & $\begin{array}{r}1,770.94 \\
18,878.50\end{array}$ & $\begin{array}{r}(6) 8,648.51 \\
11,559.51 \\
9,089.93\end{array}$ & & & \\
\hline$s_{3} p_{7}$ & $\begin{array}{c}\lambda \\
\nu \\
m p_{7}\end{array}$ & $\begin{array}{r}2,759.24 \\
18,878.50\end{array}$ & $\begin{array}{r}(10) 7,967.33 \\
12,547.81 \\
9,089.93\end{array}$ & & & \\
\hline$s_{4} p_{7}$ & $\begin{array}{c}\lambda \\
\nu \\
m p_{7}\end{array}$ & $\begin{array}{r}(40) 9,162.66 \\
10,910.87 \\
18,878.47\end{array}$ & $\begin{array}{r}(70) 4,829.71 \\
20,699.41 \\
9,089.93\end{array}$ & $\begin{array}{r}(15) 4,116.11 \\
24,287.95 \\
5,501.39\end{array}$ & $\begin{array}{r}(3) 3,826.86 \\
26,123.70 \\
3,665.64\end{array}$ & $\begin{array}{r}(1) 3,679.31 \\
27,171.30 \\
2,618.04\end{array}$ \\
\hline$s_{5} p_{7}$ & $\begin{array}{c}\lambda \\
\nu \\
m p 7\end{array}$ & $\begin{array}{r}(60) 8,409.21 \\
11,888.46 \\
18,878.52\end{array}$ & $\begin{array}{r}(8) 4,611.88 \\
21,677.08 \\
9,089.90\end{array}$ & $\begin{array}{r}(1) 3,956.88 \\
25,265.31 \\
5,501.67\end{array}$ & & \\
\hline Mean & $m p_{7}$ & $18,878.50$ & $9,089.92$ & $5,501.53$ & $3,665.64$ & $2,618.04$ \\
\hline & $\sqrt{\frac{R}{m p_{7}}}$ & 2.4110 & 3.4743 & 4. 4661 & 5. 4714 & 6.4742 \\
\hline
\end{tabular}


$\mathrm{T}_{\mathrm{ABLE}}$ 5.-Xe principal series, $1 s_{\mathrm{i}}-m_{\mathrm{k}}-$ Continued

Series, $m p_{8}$

Limit: $1 s_{5}=30,766.98$

\begin{tabular}{|c|c|c|c|c|c|c|c|}
\hline Comb. & $m$ & 2 & 3 & 4 & 5 & 6 & 7 \\
\hline$s_{5} p_{8}$ & $\begin{array}{l}\lambda \\
\nu \\
m p_{8}\end{array}$ & $\begin{array}{r}(200) 8,819.44 \\
11,335.47 \\
19,431.51\end{array}$ & $\begin{array}{r}(300) 4,671.22 \\
21,401.71 \\
9,365.27\end{array}$ & $\begin{array}{r}(30) 3,967.55 \\
25,197.37 \\
5,569.61\end{array}$ & $\begin{array}{r}(5) 3,693.50 \\
27,066.92 \\
3,700.06\end{array}$ & $\begin{array}{r}(2) 3,554.03 \\
28,129.07 \\
2,637.91\end{array}$ & $\begin{array}{r}(1) 3,472.43 \\
28,790.06 \\
1,976.92\end{array}$ \\
\hline Mean & $m p_{8}$ & $19,431.51$ & $9,365.27$ & $5,569.61$ & $3,700.06$ & $2,637.91$ & $1,976.92$ \\
\hline & $\sqrt{\frac{R}{m p_{8}}}$ & 2. 3764 & 3. 4231 & 4.4388 & 5.4459 & 6.4498 & 7.4504 \\
\hline
\end{tabular}

Series, $m p_{9}$

Limits: $1 s_{2}=20,649.44$

$1 s_{4}=29,789.34$

$1 s_{5}=30,766.98$

\begin{tabular}{|c|c|c|c|c|c|}
\hline Comb. & $m$ & 2 & 3 & 4 & 5 \\
\hline$s_{2} p_{\theta}$ & $\stackrel{\lambda}{\nu}$ & $\begin{array}{r}934.72 \\
19,714.72\end{array}$ & $\begin{array}{r}(50) 8,952.25 \\
11,167.31 \\
9,482.13\end{array}$ & & \\
\hline$s_{4} p_{0}$ & $\begin{array}{c}\lambda \\
\nu \\
m p_{9}\end{array}$ & $\begin{array}{r}\text { (10) } 9,923.10 \\
10,074.74 \\
19,714.60\end{array}$ & $\begin{array}{r}(60) 4,923.15 \\
20,306.55 \\
9,482.79\end{array}$ & $\begin{array}{r}(4) 4,135.12 \\
24,176.30 \\
5,613.04\end{array}$ & \\
\hline$s_{s} p_{0}$ & $\begin{array}{c}\lambda \\
\nu \\
m p 9\end{array}$ & $\begin{array}{r}(50) 9,045.46 \\
11,052.24 \\
19,714.74\end{array}$ & $\begin{array}{r}(70) 4,697.02 \\
21,284.16 \\
9,482.82\end{array}$ & $\begin{array}{r}(5) 3,974.42 \\
25,153.81 \\
5,613.17\end{array}$ & $\begin{array}{r}(1) 3,696.82 \\
27,042.61 \\
3,724.37\end{array}$ \\
\hline Mean & $m p_{9}$ & $19,714.72$ & $9,482.80$ & $5,613.1$ & $3,724.37$ \\
\hline . & $\sqrt{\frac{R}{m n^{n}}}$ & 2. 3593 & 3.4018 & 4. 4216 & 5.4281 \\
\hline
\end{tabular}

Series, $m p_{10}$

Limits: $1 s_{2}=20,649.44$

$1 s_{3}=21,637.74$

$1 s_{4}=29,789.34$

$1 s_{5}=30,766.98$

\begin{tabular}{|c|c|c|c|c|c|}
\hline Comb. & $m$ & 2 & 3 & 4 & 5 \\
\hline$s_{2} p_{10}$ & $\begin{array}{l}\lambda \\
\stackrel{\nu}{m} p_{10}\end{array}$ & $\begin{array}{r}84.11 \\
20,565.33\end{array}$ & $\begin{array}{r}(1) 9,306.56 \\
10,724.17 \\
9,907.27\end{array}$ & $\begin{array}{r}(4) 6,678.94 \\
14,968.31 \\
5,681.13\end{array}$ & $\begin{array}{r}(1) 5,921.79 \\
16,882.12 \\
3,767.32\end{array}$ \\
\hline$s_{3} p_{10}$ & 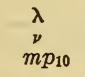 & $\begin{array}{r}1,072.41 \\
20,565.33\end{array}$ & $\begin{array}{r}(2) 8,522.49 \\
11,730.44 \\
9,907.30\end{array}$ & $\begin{array}{r}(4) 6,265.28 \\
15,956.57 \\
5,681.17\end{array}$ & $\begin{array}{r}(1) 5,59421 \\
17,870.68 \\
3,767.06\end{array}$ \\
\hline$s_{4} p_{10}$ & $\begin{array}{l}\lambda \\
\stackrel{\nu}{m p_{10}}\end{array}$ & $\begin{array}{r}9,224.01 \\
20,565.33\end{array}$ & $\begin{array}{r}(15) 5,028.26 \\
19,882.07 \\
9,907.27\end{array}$ & & \\
\hline$s_{5} p_{10}$ & $\begin{array}{l}\lambda \\
\stackrel{\nu}{m p_{10}}\end{array}$ & $\begin{array}{r}(20) 9,799.65 \\
10,201.65 \\
20,565.33\end{array}$ & $\begin{array}{r}(20) 4,792.61 \\
20,859.64 \\
9,907.34\end{array}$ & $\begin{array}{r}(5) 3,985.22 \\
25,085.65 \\
5,681.33\end{array}$ & $\begin{array}{r}(1) 3,702.72 \\
26,999.52 \\
3,767.46\end{array}$ \\
\hline Mean & $m p_{10}$ & $20,565.33$ & $9,907.29$ & $5,681.21$ & $3,767.26$ \\
\hline & $\sqrt{\frac{R}{m p_{10}}}$ & 2. 3100 & 3. 3281 & - 4.3950 & 5. 3972 \\
\hline
\end{tabular}

From Table 5 it can readily be seen that the 10 principal series, $1 s_{1}-m p_{\mathrm{k}}$, are separated into two groups, $p_{1,2,3,4}$ going to the higher level ${ }^{2} \mathrm{P}_{1}$ of the ion, and $p_{5,6,7,8,9,10}$ to the lower level ${ }^{2} \mathrm{P}_{2}$. In the $\mathrm{Kr}_{\mathrm{I}}$ analysis we were able to establish two members of the non$73113^{\circ}-29-9$ 
Ritzian series, but in $\mathrm{Xe}_{\mathrm{I}}$ only the first members can be obtained, since, on account of the very large displacement constant $A$, the second members will have negative term values.

No lines of the principal series can be expected shorter than $3249 \mathrm{~A}$, and between this limit and the resonance line at $1469 \mathrm{~A}$, no $\mathrm{Xe}_{\mathrm{I}}$ lines can be produced. The longest wave length of the principal series in the $\mathrm{Xe}_{\mathrm{I}}$ spectrum is given by the combination $1 s_{2}-2 p_{10}=84.11$, corresponding to a wave length of $1,188,919 \mathrm{~A}=118.8919 \mu=1 / 8 \mathrm{~mm}$ (approximately).

\section{THE "FUNDAMENTAL" SERIES ( $\mathrm{X}, \mathrm{Y}, Z, \mathrm{Z}, \mathrm{V}$, AND W)}

Paschen found two unidentified terms, $x$ and $y$, in the $\mathrm{Ne}_{\mathrm{I}}$ spectrum and when Messiner found three similar terms in the $A_{I}$ spectrum, he called them $X, Y$, and $Z$. Meissner ${ }^{30}$ pointed out that these series begin with order number $m=4$, are hydrogen-like, and might, therefore, be regarded as $f$-terms. Since, however, these terms combined with $s$ - and $d$-terms but not with $p$-terms, the simple selection rule $\Delta l= \pm 1$ was violated and it was necessary to hold in reserve the final interpretation of these $f$-type terms.

The authors also found two series, $X$ and $Y$, in the $\mathrm{Kr}_{\mathrm{I}}$ spectrum, and interpreted them as terms arising from the configuration $\left(s^{2} p^{5}\right) f$. We now feel certain that this is the correct explanation, because we have found this term group much more fully developed in the $\mathrm{Xe}_{\mathrm{I}}$ spectrum. In fact, 6 series and 34 terms belonging to the $\left(s^{2} p^{5}\right) f$ configuration have been detected in $\mathrm{Xe}_{\mathrm{I}}$; the $X$ and $Y$ series combinations with $s$-terms are presented in Table 6 .

\section{TABLE 6. -Xe fundamental series, $1 s_{\mathrm{i}}-m f_{\mathrm{k}}$}

SERIES, $m X$

Limits: $1 s_{2}=20,649.44$

$1 s_{3}=21,637.74$

$1 s_{4}=29,789.34$

$1 s_{5}=30,766.98$

\begin{tabular}{|c|c|c|c|c|c|c|c|}
\hline Comb. & $m$ & 4 & 5 & 6 & $y$ & 8 & 9 \\
\hline$s_{2} X$ & $\left\{\begin{array}{c}\lambda \\
{ }_{m}^{\nu} X\end{array}\right.$ & $\begin{array}{r}(5) 7,321.46 \\
13,654.73 \\
6,994.71\end{array}$ & $\begin{array}{r}\text { (7) } 6,179.66 \\
16,177.65 \\
4,471.79\end{array}$ & $\begin{array}{r}\text { (3) } 5,696.44 \\
17,549.96 \\
3,099.48\end{array}$ & $\begin{array}{r}\text { (1) } 5,440.2 \\
18,376.58 \\
2,272.86\end{array}$ & $\begin{array}{r}\text { (1) } 5,286.1 \\
18,912.29 \\
1,737.15\end{array}$ & $\begin{array}{r}(1-) 5,185.33 \\
19,279.82 \\
1,369.62\end{array}$ \\
\hline$s_{3} X$ & $\left\{\begin{array}{c}\lambda \\
\stackrel{\nu}{m X}\end{array}\right.$ & $\begin{array}{r}(40) 6,827.30 \\
14,643.04 \\
6,994.70\end{array}$ & $\begin{array}{r}\text { (20) } 5,823.89 \\
17,165.91 \\
4,471.83\end{array}$ & $\begin{array}{r}\text { (4) } 5,392.74 \\
18,538.31 \\
3,099.43\end{array}$ & $\begin{array}{r}\text { (2) } 5,162.66 \\
19,364.48 \\
2,273.26\end{array}$ & $\begin{array}{r}(1) 5,023.84 \\
19,899.56 \\
1,738.18\end{array}$ & \\
\hline$s_{4} X$ & $\left\{\begin{array}{c}\lambda \\
\nu \\
m X\end{array}\right.$ & $\begin{array}{r}(10) 4,385.75 \\
22,794.73 \\
6,994.61\end{array}$ & $\begin{array}{r}(1) 3,948.70 \\
25,317.65 \\
4,471.69\end{array}$ & & & & \\
\hline$s_{5} X$ & $\left\{\begin{array}{c}\lambda \\
\stackrel{\nu}{m X}\end{array}\right.$ & $\begin{array}{r}(3) 4,205.37 \\
23,772.45 \\
6,994.53\end{array}$ & & & & & \\
\hline Mean & $m X$ & $6,994.68$ & $4,471.82$ & $3,099.40$ & $2,273.04$ & $1,737.83$ & $1,369.62$ \\
\hline & $\sqrt{\frac{R}{m \bar{X}}}$ & 3. 9609 & 4. 9538 & 5.9503 & 6.9482 & 7.9464 & 8.9510 \\
\hline
\end{tabular}

${ }^{30}$ Meissner, Zeit. f. Phys., 39, p. 178; 1926. 
TABLE 6.-X $-X e_{\mathrm{I}}$ fundamental series, $1 s_{\mathrm{i}}-m f_{\mathrm{k}}$-Continued

\section{SERIES $m Y$}

Limits: $1 s_{2}=20,649.44$

$1 s_{4}=29,789.34$

$1 s_{5}=30,766.98$

\begin{tabular}{|c|c|c|c|c|c|c|c|}
\hline Comb. & $m$ & 4 & 5 & 6 & $\boldsymbol{y}$ & 8 & 9 \\
\hline $8_{2} Y$ & $\stackrel{\lambda}{\nu}$ & $\begin{array}{r}\text { (5) } 7,316.28 \\
13,664.39 \\
6,985.05\end{array}$ & $\begin{array}{r}(9) 6,178.30 \\
16,181.21 \\
4,468.23\end{array}$ & $\begin{array}{r}(5) 5,695.74 \\
17,552.12 \\
3,097.32\end{array}$ & $\begin{array}{r}\text { (1) } 5,440.2 \\
18,377.60 \\
2,271.84\end{array}$ & $\begin{array}{r}\text { (1) } 5,286.1 \\
18,912.29 \\
1,737.15\end{array}$ & $\begin{array}{r}(1-) 5,185.33 \\
19,279.82 \\
1,369.62\end{array}$ \\
\hline$s_{4} Y$ & $\begin{array}{c}\lambda \\
\nu \\
m Y\end{array}$ & $\begin{array}{r}(15) 4,383.90 \\
22,804.35 \\
6,984.99\end{array}$ & $\begin{array}{r}(5) 3,948.19 \\
25,320.92 \\
4,468.42\end{array}$ & $\begin{array}{r}\text { (2) } 3,745.44 \\
26,691.57 \\
3,097.77\end{array}$ & $\begin{array}{r}\text { (2) } 3,633.15 \\
27,516.51 \\
2,272.83\end{array}$ & & \\
\hline$s_{5} Y$ & $\begin{array}{c}\lambda \\
\nu \\
m Y\end{array}$ & $\begin{array}{r}\text { (10) } 4,203.69 \\
23,781.95 \\
6,985.03\end{array}$ & $\begin{array}{r}\text { (3) } 3,801.43 \\
26,298.45 \\
4,468.53\end{array}$ & $\begin{array}{r}\text { (2) } 3,613.07 \\
27,669.43 \\
3,097.55\end{array}$ & & & \\
\hline Mean & $m Y$ & $6,985.03$ & $4,468.35$ & $3,097.50$ & $2,272.14$ & $1,737.15$ & $1,369.62$ \\
\hline & $\frac{R}{m Y}$ & 3.9636 & 4. 9557 & 5. 9521 & 6.9496 & 7.9480 & 8.9510 \\
\hline
\end{tabular}

These $X$ and $Y$ series are hydrogenlike in every case and may, therefore, be called "fundamental" to distinguish them from "principal" and "subordinate" series. The terms of the $f$-series have nearly the same value in different rare-gas spectra, tending to increase slightly with heavier atoms. The first members of the $X$ and $Y$ series (and effective quantum numbers) in different spectra are compared in Table 7, to which is added a comparison of the values for the $3 d_{4}{ }^{\prime}$ term. It is seen that the $12 d$-terms arising from the $\left(s^{2} p^{5}\right) 3 d$ configuration assume a relatively deeper position in the energy diagram as we pass from lighter to heavier atoms. This is exemplified by the increase of $3 d_{4}{ }^{\prime}$ from 12,339 in $\mathrm{Ne}_{\mathrm{I}}$ to 17,636 in $\mathrm{Xe}_{\mathrm{I}}$. The $m d-m f$ combinations in $\mathrm{Ne}_{\mathrm{I}}, \mathrm{A}_{\mathrm{I}}$, and $\mathrm{Kr}_{\mathrm{I}}$ fall in the infra-red outside of the range of photographic investigation, but in $\mathrm{Xe}_{\mathrm{I}}$, on account of the larger interval between the $d$ - and $f$-terms, it was possible to observe the $3 d-m f$ combinations. This explains why we observed about 50 per cent more lines in $\mathrm{Xe}_{\mathrm{I}}$ than in $\mathrm{Kr}_{\mathrm{I}}$ although essentially the same procedure was used in both. These combinations may be expected to be even more prominent in the spectrum of Radon.

TABLE 7.-Comparison of $d$-and f-terms in rare-gas spectra

\begin{tabular}{|c|c|c|c|c|c|}
\hline & $\mathrm{Ne}_{\boldsymbol{x}}$ & $A_{X}$ & $\mathrm{Kr}_{x}$ & $X e_{x}$ & $\mathrm{Rn}_{\mathrm{I}}$ \\
\hline $\begin{array}{l}4 X\left(f_{6}\right) \\
n_{\text {eff }} \\
4 Y^{\text {eff }}\left(f_{5}\right) \\
n_{\text {eff }}\end{array}$ & $\begin{array}{r}6,876.73 \\
3.9940 \\
6,860.23 \\
3.9988\end{array}$ & $\begin{array}{r}6,922.91 \\
3.9813 \\
6,881.55 \\
3.9933\end{array}$ & $\begin{array}{r}6,950.21 \\
3.9726 \\
6,893.68 \\
3.9896\end{array}$ & $\begin{array}{r}6,994.68 \\
3.9609 \\
6,985.03 \\
3.9636\end{array}$ & $\begin{array}{c}(7,000 \pm) \\
? \\
(7,000 \pm) \\
?\end{array}$ \\
\hline $\begin{array}{l}3 d_{4}{ }^{\prime} \\
n_{\text {eff }}\end{array}$ & $\begin{array}{r}12,339.15 \\
2.9816\end{array}$ & $\begin{aligned} 14, & 362.0 \\
& 2.7642\end{aligned}$ & $\begin{array}{r}(15,400 \pm) \\
(2.6694)\end{array}$ & $\begin{array}{r}17,636.85 \\
2.4944\end{array}$ & $\begin{array}{c}(20,000 \pm) \\
?\end{array}$ \\
\hline
\end{tabular}


It is quite probable that some of the unidentified $A_{I}$ and $K r_{I}$ lines in the infra-red are combinations of this type. The $\left(s^{2} p^{5}\right) f$ terms combine with the terms arising from $\left(s^{2} p^{5}\right) 1 s$ and from $\left(s^{2} p^{5}\right) 3 d$ as shown in Table 8. These combinations, as well as the analogous ones in the $\mathrm{Ne}_{\mathrm{I}}, \mathrm{A}_{\mathrm{I}}$ and $\mathrm{Kr}_{\mathrm{I}}$ spectra, are in perfect accord with the revised rules governing transitions when two or more electrons are involved. ${ }^{31}$ Transitions occur for which the $l$ of one electron changes \pm 1 while the $l$ of another changes 0 or \pm 2 . In other words, transitions occur only when the sum of all changes in $l$ is odd. Thus, the $s f$ combinations may be interpreted as double electron jumps in which the $s$ electron makes a transit to a $p$ orbit $(\Delta l=1)$ while the $p$ electron is passing to an $f$ orbit $(\Delta l=2)$.

The series $3 d_{4}{ }^{\prime}-m Z$ and $3 d_{1}{ }^{\prime \prime}-m Z$ can be used for the calculation of the term values $3 d_{4}{ }^{\prime}$ and $3 d_{1}{ }^{\prime \prime}$, not obtainable in any other way. The values thus derived are $3 d_{4}{ }^{\prime}=17,637.11$ and $3 d_{1}{ }^{\prime \prime}=16,863.45$. This permits the exact calculation of an important line in the far infra-red, namely, $2 p_{8}-3 d_{4}{ }^{\prime}$ (see Table 9). The wave number of this line is $\nu=18,431.77-$ $17,637.11=1,794.66$, and the wave length $5.562 \mu$. An approximate value for this line can be obtained by extrapolating the $2 p_{8}-m d_{4}^{\prime}$ series to its first member. This procedure gives $\nu=2 p_{8}-3 d_{4}{ }^{\prime}=$ 1,809.64; which compares favorably with the exact calculation above, remembering that the approximation could be expected to yield too large a value since the calculated second member $\nu_{\text {calc. }}=10,511.37$ is somewhat larger than the observed $\nu_{\text {obs. }}=10,508.71$.

Furthermore, it is now possible to calculate the leading member of the $2 p_{9}-3 d_{4}$ series in the infra-red; $2 p_{9}-3 d_{4}=19,714.98-17,512.15=$ $2,202.84 ; \lambda=4.5396 \mu$.

31 Hund, Linienspektren und periodisches System der Elemente, p. 132, Julius Springer, Berlin; 1927. 


\begin{tabular}{|c|c|c|c|c|c|c|}
\hline & $\begin{array}{l}\text { AN } \\
\text { E. }\end{array}$ & $\stackrel{\lambda}{\text { E. }}$ & № & $\begin{array}{l}\text { DD } \\
\text { हn. }\end{array}$ & $\begin{array}{l}\vec{B} \\
\mathrm{E}\end{array}$ & E. \\
\hline & 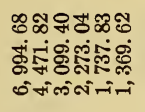 & 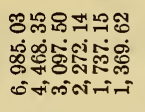 & 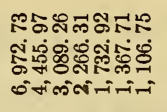 & 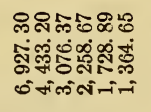 & 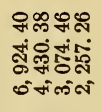 & 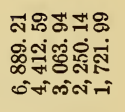 \\
\hline $\begin{array}{r}\infty \\
\infty \\
0 \\
0 \\
0 \\
0 \\
0\end{array}$ & 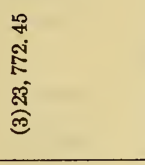 & 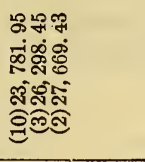 & & 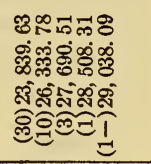 & 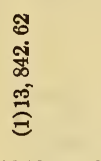 & \\
\hline 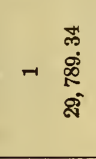 & 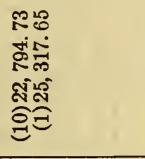 & 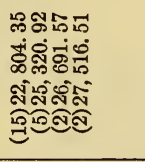 & & & 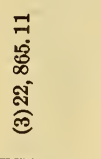 & \\
\hline 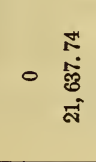 & 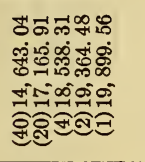 & & $\stackrel{I}{=}$ & & & \\
\hline 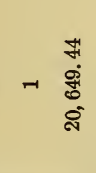 & 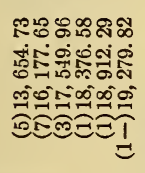 & 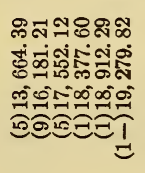 & & & 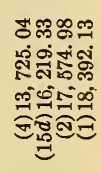 & \\
\hline 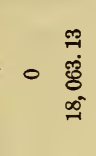 & 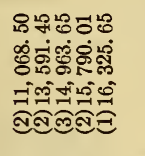 & & & & & \\
\hline 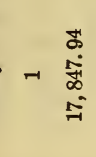 & 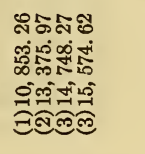 & 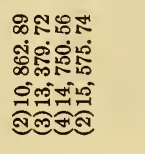 & & & 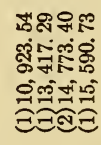 & \\
\hline 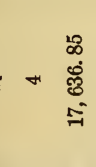 & & & 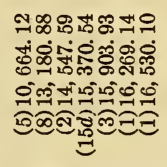 & & tr & 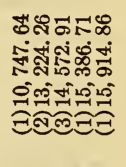 \\
\hline 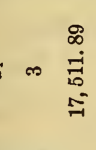 & & 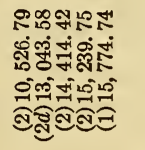 & & 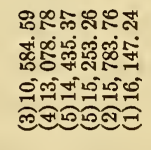 & 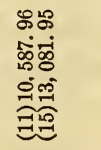 & \\
\hline 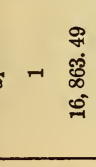 & & & 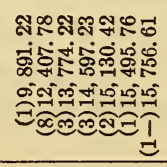 & 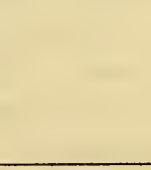 & & 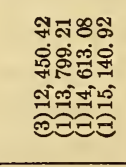 \\
\hline$\varepsilon$ & HLONDO & Hoon 000 & HoON $\infty 00$ & H20 ONDO & Hon ON & HLO ON $\infty$ \\
\hline
\end{tabular}


TABLE 9.-Determination of $3 d_{4}^{\prime}$ and $B d_{2}{ }^{\prime \prime}$

Series, $3 d_{4}{ }^{\prime}-m Z$

$$
\begin{gathered}
\nu=A-\frac{R}{[m+\mu+a(A-\nu)]^{2}} \\
R_{X_{\Theta}}=109,736.66 \\
A=17,637.112 \\
a=+0.000001918490443 \\
\mu=+0.9538523722 \pm 10^{-10}
\end{gathered}
$$

\begin{tabular}{|c|c|c|c|c|c|}
\hline$M$ & Int. & \multicolumn{1}{|c|}{$\lambda_{\text {obs. }}$} & \multicolumn{1}{|c|}{$\nu_{\text {obs. }}$} & \multicolumn{1}{c|}{$\nu_{\text {oalo. }}$} & \multicolumn{1}{c|}{$\begin{array}{c}\nu_{\text {obs. }}- \\
\nu_{\text {oalo. }}\end{array}$} \\
\hline 3 & 5 & $9,374.67$ & $10,664.12$ & $10,664.80$ & -0.68 \\
4 & 8 & $7,584.66$ & $13,180.88$ & $13,180.88$ & 0.00 \\
5 & 20 & $6,872.10$ & $14,547.59$ & $14,547.59$ & 0.00 \\
6 & $15 d$ & $6,504.16$ & $15,370.54$ & $15,370.60$ & -0.06 \\
7 & 3 & $6,286.02$ & $15,903.93$ & $15,903.97$ & -0.04 \\
8 & 1 & $6,144.91$ & $16,269.14$ & $16,269.14$ & 0.00 \\
9 & $1-$ & $6,047.9$ & $16,530.10$ & $16,529.93$ & +0.17 \\
\hline
\end{tabular}

$$
\begin{gathered}
3 d_{4}{ }^{\prime}=17,637.11 \\
\text { Series, } 3 d_{2}{ }^{\prime \prime}-m Z \\
A=16,863.453 \\
a=+0.000001943114582 \\
\mu=+0.954056552 \pm 10^{-10}
\end{gathered}
$$

\begin{tabular}{|c|c|c|c|r|r|}
\hline$M$ & Int. & \multicolumn{1}{|c|}{$\lambda_{\text {obs. }}$} & \multicolumn{1}{|c|}{$\nu_{\text {obs. }}$} & \multicolumn{1}{|c|}{$\nu_{\text {calo. }}$} & \multicolumn{1}{c|}{$\begin{array}{c}\nu_{\text {obs. }}- \\
\nu_{\text {oalo. }}\end{array}$} \\
\hline & 1 & $10,107.2$ & $9,891.22$ & $9,892.45$ & -1.23 \\
3 & 8 & $8,057.25$ & $12,407.78$ & $12,407.78$ & 0.00 \\
4 & 3 & $7,257.94$ & $13,774.22$ & $13,774.22$ & 0.00 \\
5 & 3 & $6,848.73$ & $14,597.23$ & $14,597.11$ & +0.12 \\
6 & 2 & $6,607.38$ & $15,130.42$ & $15,130.42$ & 0.00 \\
7 & 1 & $6,451.6$ & $15,495.76$ & $15,495.55$ & +0.21 \\
8 & $1-$ & $6,344.79$ & $15,756.61$ & $15,756.41$ & +0.20 \\
9 & 1 & & & \\
\hline
\end{tabular}

$$
3 d_{2}{ }^{\prime \prime}=16,863.45
$$

\section{THE SUBORDINATE SERIES}

All the observed combinations of $m s_{1}$ and $m d_{1}$ levels with the $m p_{\mathrm{s}}-$ terms are collected in Table 10. It is seen from this table that some of the combinations which can be expected on the basis of assigned inner quantum numbers have not been observed, some on account of faintness, and others because they lie in the unexplored infra-red. Especially the combinations with the non-Ritzian group $2 p_{1,2,3,4}$ lie far outside the range of photographic investigations. Radiometric investigations of the infra-red spectra of both krypton and xenon are desirable and important. 


\begin{tabular}{|c|c|c|c|c|c|c|c|}
\hline & & & है- & हొڤ & छี.॥ & = & हี స్- \\
\hline & & & 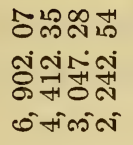 & 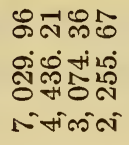 & 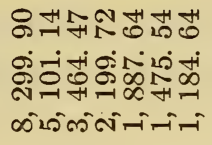 & 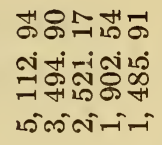 & 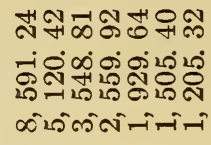 \\
\hline$\stackrel{2}{2}$ & -1 & 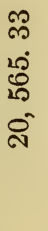 & 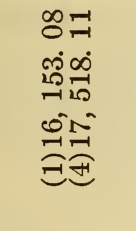 & 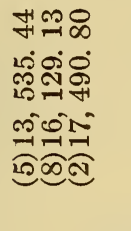 & & 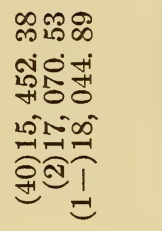 & 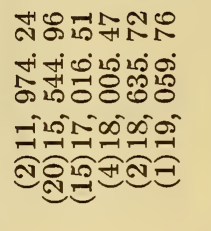 \\
\hline$\Sigma$ & N & 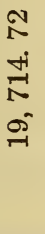 & 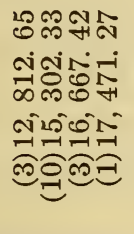 & 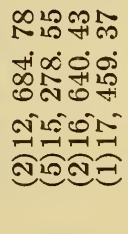 & 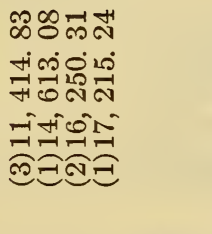 & 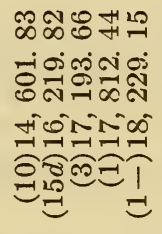 & 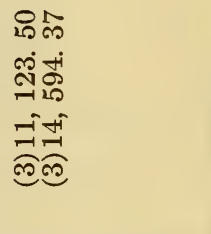 \\
\hline$\stackrel{\infty}{2}$ & $\infty$ & 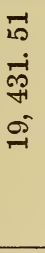 & & 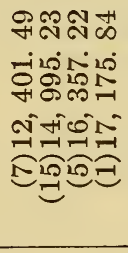 & 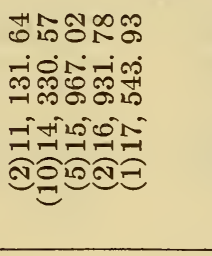 & 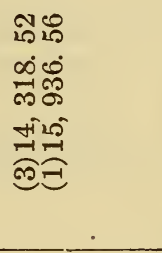 & . \\
\hline$\Sigma$ & -1 & $\begin{array}{l}\infty \\
\infty \\
\infty \\
\infty \\
\infty \\
\infty \\
\infty \\
\rightarrow-1\end{array}$ & 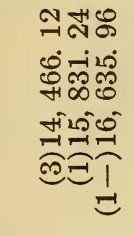 & $\begin{array}{l}\stackrel{0}{+1} \\
\infty \\
+1 \\
\infty \\
=-1 \\
\stackrel{-1}{\Xi}\end{array}$ & & 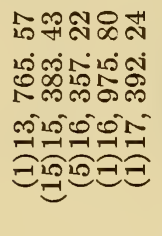 & 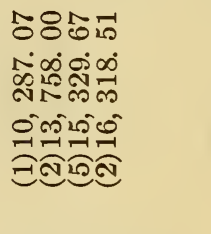 \\
\hline ฉ & N & $\begin{array}{l}\text { ठ } \\
\text { నิ } \\
0 \\
\infty \\
\infty\end{array}$ & 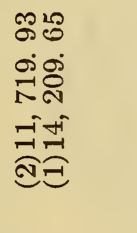 & 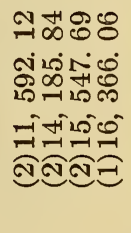 & 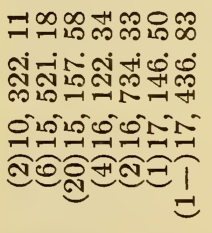 & 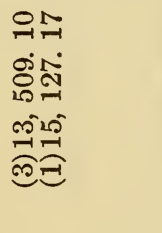 & 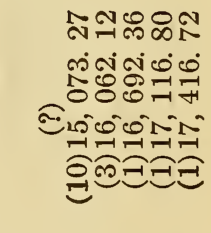 \\
\hline & 0 & & & & & & \\
\hline & ミ & & $\varpi \# 200$ & $\infty+200$ & H & $2001 \infty \infty$ & H \\
\hline
\end{tabular}




\begin{tabular}{|c|c|c|c|c|c|}
\hline & & శై & ind & हี.॥ & छั. \\
\hline & & 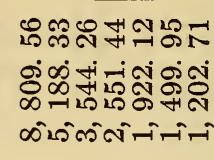 & 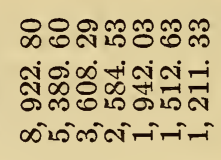 & 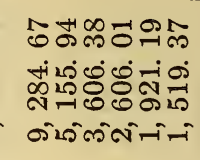 & 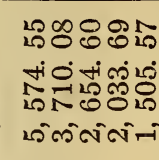 \\
\hline & $\begin{array}{r}\text { m } \\
20 \\
\text { 20 } \\
\text { 20 } \\
\text { \&ิ }\end{array}$ & & & 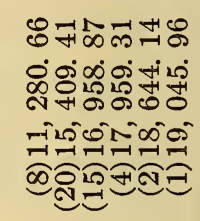 & 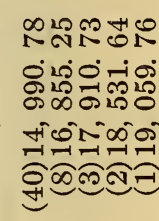 \\
\hline$\approx$ & 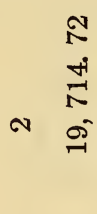 & 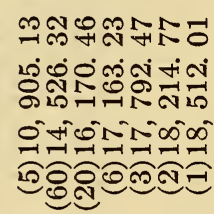 & & 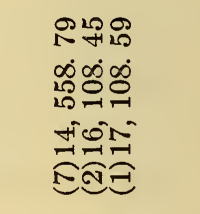 & \\
\hline$\stackrel{\infty}{\infty}$ & 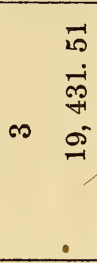 & 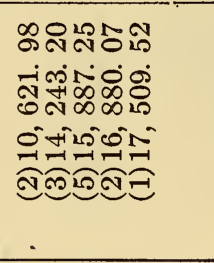 & 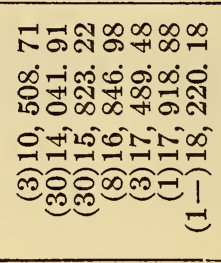 & & \\
\hline$\varepsilon$ & 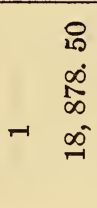 & & & 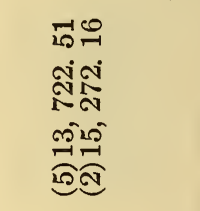 & 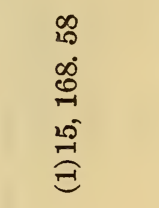 \\
\hline & 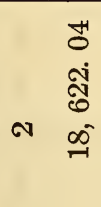 & 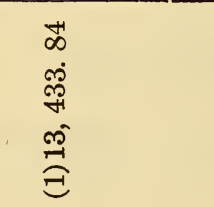 & & 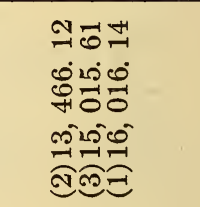 & \\
\hline & 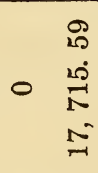 & & & & \\
\hline & $\xi$ & HLON $\infty 00$ & HLO ONDOO & H1200Nos & $200 \mathrm{~N} \infty 0$ \\
\hline
\end{tabular}


The following additional combinations (Table 11), give a plausible explanation for an outstanding group of lines in the longer waveiength region, but one or more permitted combinations are absent in each row or column.

TABLE 11.-Additional $s^{\prime} p$ combinations

\begin{tabular}{|c|c|c|c|c|c|}
\hline \multirow{3}{*}{$j$} & & $s_{1}{ }^{\prime \prime}$ & $s_{1}^{\prime \prime \prime \prime}$ & $s_{1}^{\prime \prime \prime}$ & $s_{1}^{\prime}$ \\
\hline & & 2 & 3 & $2(1)$ & 1 \\
\hline & Term & $6,387.01$ & $6,087.89$ & $5,706.16$ & $5,574.55$ \\
\hline $\begin{array}{l}1 \\
2 \\
3 \\
1 \\
2 \\
0\end{array}$ & $\begin{array}{l}p_{10} \\
p_{9} \\
p_{8} \\
p_{7} \\
p_{6} \\
p_{5}\end{array}$ & $\begin{array}{r}(2) 13,327.71 \\
(2 d) 13,043.58 \\
(3) 12,235.01\end{array}$ & $\begin{array}{l}\text { (4) } 13,626.85 \\
\text { (2) } 13,343.60\end{array}$ & $\begin{array}{l}\text { (2) } 12,915.88 \\
\text { (2) } 12,009.29\end{array}$ & (40) $14,990.78$ \\
\hline
\end{tabular}

\section{THE $\mathrm{Xe}_{\mathrm{I}}$ SPECTRUM IN THE EXTREME ULTRA-VIOLET}

The following lines in the extreme ultra-violet (Table 12) have been reported by Abbink and Dorgelo ${ }^{32}$ as characteristic of the first spectrum of xenon.

TABLE 12.-First spectrum of Xe in the extreme ultra-violet

\begin{tabular}{|c|c|c|c|c|c|}
\hline Line No. & $\begin{array}{l}\text { Inten- } \\
\text { sity }\end{array}$ & $\begin{array}{c}\text { Wave length } \\
\lambda\end{array}$ & $\underset{\nu}{\text { Wave No. }}$ & Combination & Calculated term \\
\hline $\begin{array}{l}1 \\
2 \\
3 \\
4 \\
5\end{array}$ & $\begin{array}{r}20 \\
10 \\
6 \\
5 \\
5\end{array}$ & $\begin{array}{r}1,469.61 \\
1,295.56 \\
1,277.50 \\
1,250.24 \\
1,192.02\end{array}$ & $\begin{array}{l}68,045.3 \\
77,186.7 \\
78,277.9 \\
79,984.6 \\
83,891.2\end{array}$ & $\begin{array}{l}p_{0}-1 s_{4} \\
p_{0}-1 s_{2} \\
\\
p_{0}-3 d_{5} \\
p_{0}-2 s_{4}\end{array}$ & $\begin{array}{l}29,798.0 \\
20,648.6 \\
19,557.4 \\
17,850.7 \\
13,944.1\end{array}$ \\
\hline $\begin{array}{r}6 \\
7 \\
8 \\
9 \\
10\end{array}$ & $\begin{array}{r}15 \\
10 \\
4 \\
15 \\
5\end{array}$ & $\begin{array}{r}{ }^{a} 1,100.46 \\
1,08894 \\
1,083.89 \\
1,074.50 \\
1,067.10\end{array}$ & $\begin{array}{l}90,871.1 \\
91,832.4 \\
92,260.3 \\
93,066.5 \\
93,711.9\end{array}$ & $\begin{array}{l}p_{0}-3 s_{1}{ }^{\prime} \\
p_{0}-2 s_{2}\end{array}$ & $\begin{array}{l}6,964.2 \\
6,002.9 \\
5,575.0 \\
4,768.8 \\
4,123.4\end{array}$ \\
\hline $\begin{array}{l}11 \\
12 \\
13 \\
14 \\
15\end{array}$ & $\begin{array}{r}10 \\
12 \\
8 \\
8 \\
10\end{array}$ & $\begin{array}{l}1,048.18 \\
1,041.30 \\
1,037.71 \\
1,032.51 \\
1,027.04\end{array}$ & $\begin{array}{l}95,403.5 \\
96,033.8 \\
96,366.0 \\
96,857.4 \\
97,367.2\end{array}$ & & $\begin{array}{r}2,431.8 \\
1,800.5 \\
1,469.3 \\
783.9 \\
468.1\end{array}$ \\
\hline
\end{tabular}

a Adherence to Xe doubtful.

8s Abbink and Dorgelo, Zeit. f. Phys., 47, p. 221; 1928. 
Since all true $\mathrm{Xe}_{\mathrm{I}}$ lines in the extreme ultra-violet must be combinations involving the normal level $p_{0}=97,835.3$, it is possible to calculate the combining levels, all of which must have the inner quantum number $j=1$. The first two levels give the resonance lines; they have been used for determining the normal state. The calculated level $17,850.7$ is, without doubt, our level $17,847.94$, identified as $3 d_{5}$. The level $5,575.0$ may be identified with $3 s_{1}{ }^{\prime}=5,574.55$. The levels $13,944.1$ and $4,768.8$ may be identified with $2 s_{4}$ and $2 s_{2}$, respectively, because they have a separation of $9,175.3$; the separation $1 s_{4}-1 s_{2}$ is $9,139.90$, and this term difference is expected to increase with higher terms approaching the ${ }^{2} \mathrm{P}_{2,1}$ interval, 9,621 , in the limit.

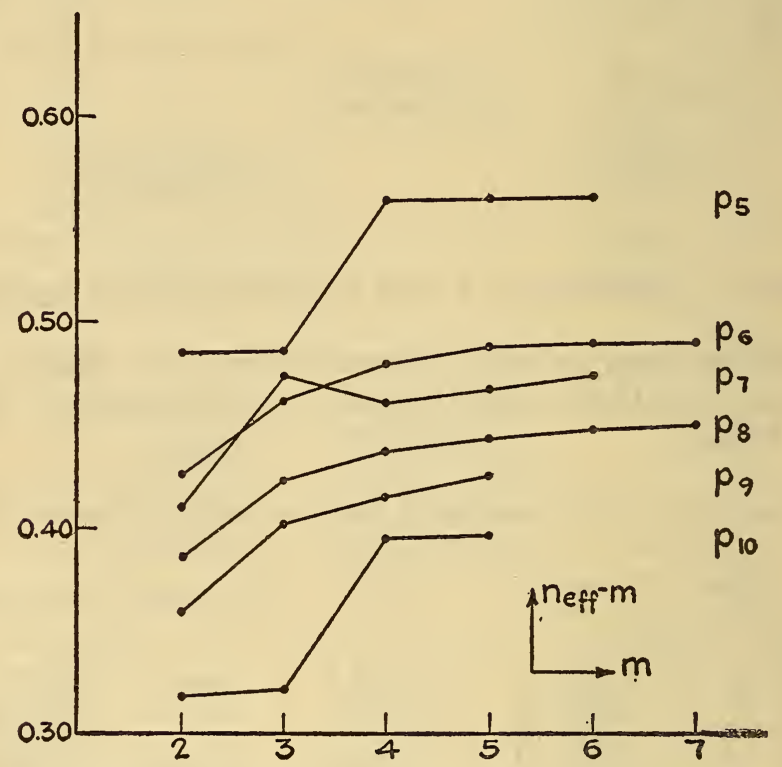

Figdre 6.-Effective quantum numbers for $p$-terms

At this point we may also refer to the $2 s_{3}$ level; it must be a little larger than $2 s_{2}$, and the combination with $2 p_{10}$ should have a w.ve number of about $15,700 \mathrm{~cm}^{-1}$. Now, the only unclassified line in this region is $\nu=15,729.52$. which is accordingly interpreted as $2 p_{10}-2 s_{3}$. The only other possible $s p$ combination is $2 p_{7}-2 s_{3} \quad\left(\nu_{\text {calc. }}=\right.$ $14,042.74)$, which unfortunately is masked by the strong line $2 p_{8}-5 d^{\prime}{ }_{4}$ ( $\left.\nu_{\text {obs. }}=14,041.91\right)$.

In the case of $\mathrm{Kr}_{\mathrm{I}}$, it was possible to account for all the extreme ultra-violet lines, but it is seen from Table 12 that a considerable number of strong lines attributed to the $\mathrm{Xe}_{\mathrm{I}}$ spectrum by Abbink and Dorgelo remains unclassified. It appears that another interpretation will have to be sought for the remaining lines. 


\section{IDENTIFICATION OF THE EXPERIMENTAL TERMS}

A complete list of the identified $\mathrm{Xe}_{\mathrm{I}}$ terms is presented in Table 13. The first column contains the type of electron which, when added to $s^{2} p^{5}$, gives rise to groups of terms shown in the second column (Paschen notation), followed by the inner quantum numbers assigned

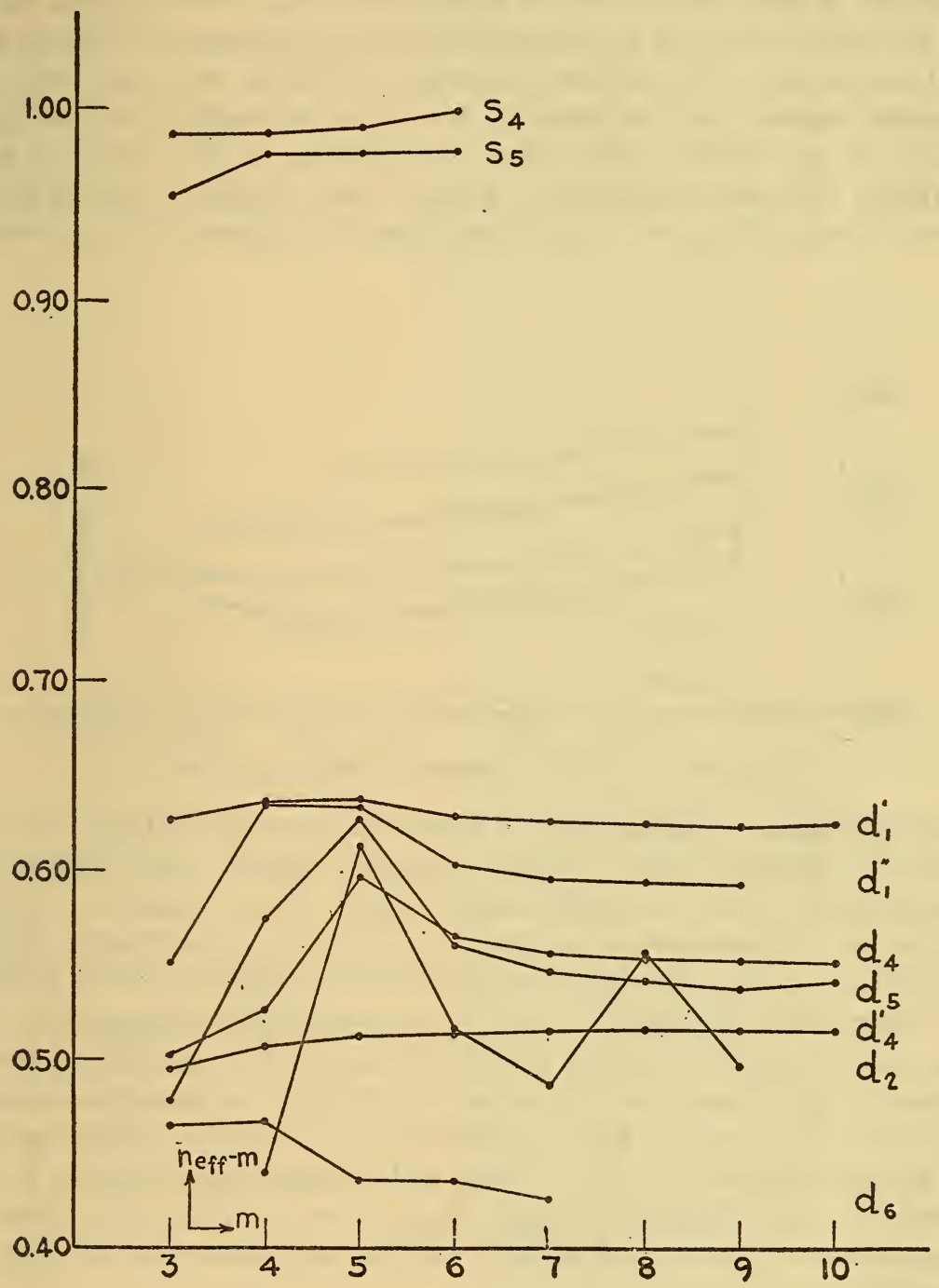

FIgURE 7.-Effective quantum numbers for $d$ - and s-terms

to the terms. The absolute value of the ground level or highest term appears in column 4 , and the remaining columns contain alternately the term values and the corresponding effective quantum numbers. The latter are an excellent criterion for arranging terms in series, and when they are plotted as a function of order number they reveal 
certain relations and regularities not otherwise obvious. Such plots are shown in Figures 6,7 , and $8, p$-terms in Figure $6, d$ - and $s^{\prime}$-terms in Figure 7, and $f$-terms in Figure 8 . The order numbers $(m)$ of the terms in the series are taken for abscissæ and the fractional part of the corresponding effective quantum numbers $\left(n_{\mathrm{eit}}-m\right)$ as ordinates. On account of the relatively small departures of $n_{\text {eff }}$ from integral values in the cases of $f$-terms an expanded scale was necessary in Figure 8.

Comparison with similar diagrams published for other rare gas spectra shows that, in general, the series converging to the upper limit ${ }^{2} \mathrm{P}_{1}$ are shorter than those coordinated to ${ }^{2} \mathrm{P}_{2}$. This is most striking with the heavier gas; whereas two members each of $p_{1,2,3,4}$ were found in $\mathrm{Kr}_{\mathrm{I}}$, only one of each can be detected in $\mathrm{Xe}_{\mathrm{I}}$ because

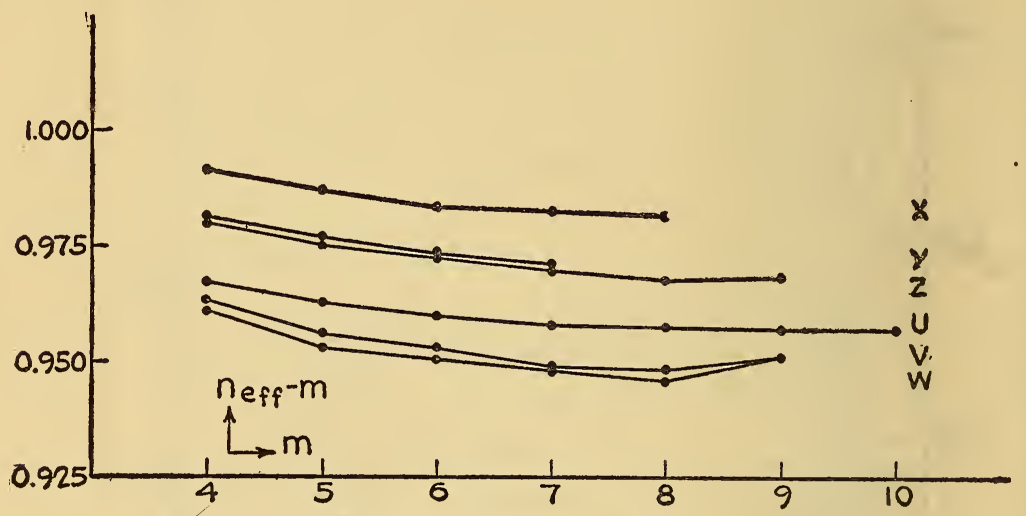

FigdRe 8.-Effective quantum numbers for f-terms

the separation of the groups of terms has become so large that the second members would acquire negative values. The behavior of homologous terms is remarkably similar in all rare gas spectra Thus, in each case certain $p$-terms $\left(p_{6}, p_{7}\right.$, and $\left.p_{8}, p_{9}\right)$ are fairly regular, whereas $p_{5}$ and $p_{10}$ are most irregular but nevertheless remain parallel.

Comparison of Figure 7 with the corresponding diagrams for $\mathrm{Ne}_{\mathrm{I}}$ $A_{I}$, and $K_{r_{I}}$ calls attention to the fact that in all cases the $d^{\prime}{ }_{4}$ series gives the most nearly perfect smooth curves, but whereas the constant $\alpha$ of the Ritz formula has a positive value in the first three spectra it becomes negative in $\mathrm{Xe}_{\mathrm{I}}$. Most of the remaining $d$-series are very irregular and dissimilar except that for $m=5$ they all appear to experience in greater or smaller degree the same perturbation or resonance.

The curves for the $f$-terms (fig. 8 ) show no large discontinuities; they behave more like the hydrogen series, as would be expected if they really involve the larger $f$-orbits. 


\section{LIST OF $\mathrm{Xe}_{\mathrm{I}}$ LINES}

A complete list of the observed lines characteristic of the first spectrum of xenon is presented in Table 14, in which the estimated intensities appear in the first column, the wave lengths (international scale) in the second, wave numbers (vacuum) in the third, and term combinations in the last. Practically all of the 318 observed lines are accounted for as combinations of established terms presented in the preceding table. Only a few lines, most of which are very faint, remain unclassified; it will perhaps be possible to place them when related, but still fainter lines have been recorded.

$\mathrm{T}_{\mathrm{ABLE}}$ 14.-List of $\mathrm{X} e_{\mathrm{I}}$ lines

\begin{tabular}{|c|c|c|c|}
\hline Intensity & Wave length & Wave number & Combination \\
\hline $\begin{array}{r}1 \\
10 \\
20 \\
1 \\
2\end{array}$ & $\begin{array}{r}10,107.2 \\
9,923.10 \\
9,799.65 \\
9,718.27 \\
9,685.29\end{array}$ & $\begin{array}{r}9,891.22 \\
10,074.74 \\
10,201.65 \\
10,287.07 \\
10,322.11\end{array}$ & $\begin{array}{l}3 d_{1}^{\prime \prime}-4 Z \\
1 s_{4}-2 p_{9} \\
1 s_{5}-2 p_{10} \\
2 p_{6}-4 d_{5} \\
2 p_{6}-4 d_{1}^{\prime}\end{array}$ \\
\hline $\begin{array}{l}6 \\
2 \\
3 \\
1- \\
2\end{array}$ & $\begin{array}{l}9,513.34 \\
9,496.97 \\
9,445.34 \\
9,442.1 \\
9,411.86\end{array}$ & $\begin{array}{l}10,508.67 \\
10,526.79 \\
10,584.37 \\
10,587.96 \\
10,621.98\end{array}$ & $\begin{array}{l}2 p_{8}-4 d_{4}{ }^{\prime} \\
3 d_{4}-4 Y \\
3 d_{4}-4 U \\
3 d_{4}-4 V \\
2 p_{8}-4 d_{4}\end{array}$ \\
\hline $\begin{array}{l}5 \\
1 \\
1 \\
1 \\
2\end{array}$ & $\begin{array}{l}9,374.67 \\
9,306.56 \\
9,301.82 \\
9,211.3 \\
9,203.13\end{array}$ & $\begin{array}{l}10,664.12 \\
10,742.17 \\
10,747.64 \\
10,853.26 \\
10,862.89\end{array}$ & $\begin{array}{c}3 d_{4}{ }^{\prime}-4 Z \\
1 s_{2}-3 p_{10} \\
3 d_{4}{ }^{\prime}-4 W \\
3 d_{5}-4 X \\
3 d_{5}-4 Y\end{array}$ \\
\hline $\begin{array}{r}5 \\
40 \\
1 \\
1 \\
50\end{array}$ & $\begin{array}{l}9,167.48 \\
9,162.66 \\
9,152.03 \\
9,095.57 \\
9,045.46\end{array}$ & $\begin{array}{l}10,905.13 \\
10,910.87 \\
10,923.54 \\
10,990.87 \\
11,052.24\end{array}$ & $\begin{array}{l}2 p_{9}-4 d_{4} \\
1 s_{4}-2 p_{7} \\
3 d_{5}-4 V \\
1 s_{5}-2 p_{9}\end{array}$ \\
\hline $\begin{array}{l}2 \\
1 \\
3 \\
2\end{array}$ & $\begin{array}{l}9,032.17 \\
9,025.74 \\
8,987.51 \\
8,980.94\end{array}$ & $\begin{array}{l}11,068.50 \\
11,076.38 \\
11,123.50 \\
11,131.64\end{array}$ & $\begin{array}{l}3 d_{6}-4 X \\
2 p_{9}-4 d_{5} \\
2 p_{8}-4 d_{1}^{\prime}\end{array}$ \\
\hline 50 & $8,952.25$ & $11,167.31$ & $\left\{\begin{array}{l}1 s_{2}-3 p_{9} \\
1 s_{4}-2 p_{6}\end{array}\right.$ \\
\hline $\begin{array}{r}5 \\
1 \\
4 \\
8 \\
200\end{array}$ & $\begin{array}{l}8,930.81 \\
8,913.33 \\
8,908.70 \\
8,862.30 \\
8,819.44\end{array}$ & $\begin{array}{l}11,194.03 \\
11,216.08 \\
11,221.91 \\
11,280.66 \\
11,335.47\end{array}$ & $\begin{array}{r}1 s_{3}-2 p_{4} \\
\\
2 p_{10}-4 d_{2} \\
1 s_{5}-2 p_{8}\end{array}$ \\
\hline $\begin{array}{r}3 \\
10 \\
1 \\
1 \\
3\end{array}$ & $\begin{array}{l}8,758.13 \\
8,739.36 \\
8,725.4 \\
8,709.6 \\
8,696.80\end{array}$ & $\begin{array}{l}11,414.83 \\
11,439.35 \\
11,457.65 \\
11,478.43 \\
11,495.33\end{array}$ & $\begin{array}{l}2 p_{9}-4 d_{1}{ }^{\prime} \\
2 p_{10}-4 d_{6} \\
3 d_{1}{ }^{\prime}-5 V ? \\
3 d_{1}{ }^{\prime}-5 W\end{array}$ \\
\hline $\begin{array}{l}3 \\
6 \\
2 \\
5 \\
2\end{array}$ & $\begin{array}{l}8,692.08 \\
8,648.51 \\
8,624.18 \\
8,576.03 \\
8,530.13\end{array}$ & $\begin{array}{l}11,501.57 \\
11,559.51 \\
11,592.12 \\
11,657.21 \\
11,719.93\end{array}$ & $\begin{array}{c}1 s_{3}-3 p_{6} \\
1 s_{2}-3 p_{7} \\
2 p_{6}-3 s_{5} \\
1 s_{2}-3 p_{5} \\
2 p_{6}-3 s_{4}\end{array}$ \\
\hline
\end{tabular}


TABLE 14.-List of $X e_{\mathrm{I}}$ lines-Continued

\begin{tabular}{|c|c|c|c|}
\hline Intensity & Wave length & Wave number - & Combination \\
\hline $\begin{array}{r}2 \\
1 \\
60 \\
1 \\
1\end{array}$ & $\begin{array}{l}8,522.49 \\
8,437.6 \\
8,409.21 \\
8,392.4 \\
8,368.4\end{array}$ & $\begin{array}{l}11,730.44 \\
11,848.46 \\
11,888.46 \\
11,912.27 \\
11,946.44\end{array}$ & $\begin{array}{l}1 s_{3}-3 p_{10} \\
2 p_{7}-3 s_{5} \\
1 s_{5}-2 p_{7}\end{array}$ \\
\hline 2 & $8,348.97$ & $11,974.24$ & $\left\{\begin{array}{c}2 p_{7}-3 s_{4} \\
2 p_{1}-4 d\end{array}\right.$ \\
\hline $\begin{array}{r}50 \\
2 \\
2 \\
150\end{array}$ & $\begin{array}{l}8,346.86 \\
8,324.6 \\
8,319.46 \\
8,280.16\end{array}$ & $\begin{array}{l}\text { 11, 977. } 26 \\
12,009.26 \\
12,016.71 \\
12,073.75\end{array}$ & $\begin{array}{l}1 s_{2}-2 p_{3} \\
2 p_{5}-s_{1}{ }^{\prime \prime \prime} \\
1 s_{4}-2 p_{5}\end{array}$ \\
\hline $\begin{array}{c}20 \\
1 \\
300 \\
1 ? \\
25\end{array}$ & $\begin{array}{l}8,266.53 \\
8,258.0 \\
8,231.64 \\
8,228.7 \\
8,206.34\end{array}$ & $\begin{array}{l}12,093.65 \\
12,106.15 \\
12,144.92 \\
12,149.25 \\
12,182.36\end{array}$ & $\begin{array}{l}1 s_{2}-2 p_{2} \\
1 s_{5}-2 p_{6} \\
1 \dot{s_{3}}-2 p_{4}\end{array}$ \\
\hline $\begin{array}{l}1 \\
3 \\
2 \\
2 \\
7\end{array}$ & $\begin{array}{l}8,191.7 \\
8,171.02 \\
8,143.8 \\
8,101.95 \\
8,061.33\end{array}$ & $\begin{array}{l}12,204.13 \\
12,235.01 \\
12,275.91 \\
12,339.32 \\
12,401.49\end{array}$ & $3 d_{5}-4 p_{9}$ \\
\hline $\begin{array}{r}8 \\
3 \\
10 \\
1 \\
8\end{array}$ & $\begin{array}{l}8,057.25 \\
8,029.65 \\
7,967.33 \\
7,937.47 \\
7,887.38\end{array}$ & $\begin{array}{l}12,407.78 \\
12,450.42 \\
12,547.81 \\
12,595.02 \\
12,675.00\end{array}$ & $\begin{array}{r}3 d_{1}{ }^{\prime \prime}-5 Z \\
3 d_{1}^{\prime \prime}-5 W \\
1 s_{3}-3 p_{7} \\
2 p_{5}-5 d_{5} \\
1 s_{2}-2 p_{1}\end{array}$ \\
\hline $\begin{array}{l}2 \\
3 \\
2 \\
2\end{array}$ & $\begin{array}{l}7,881.30 \\
7,802.64 \\
7,783.63 \\
7,740.28\end{array}$ & $\begin{array}{l}12,684.78 \\
12,812.65 \\
12,843.94 \\
12,915.88\end{array}$ & $\begin{array}{c}2 p_{9}-3 s_{5} \\
2 p_{9}-3 s_{4} \\
3 d_{1}^{\prime}-6 W^{\prime} \\
2 p_{6}-s_{1}{ }^{\prime \prime \prime}\end{array}$ \\
\hline $2 d$ & $7,664.5$ & $13,043.58$ & $\left\{\begin{array}{l}3 d_{4}-5 Y \\
2 p_{8}-s_{1}{ }^{\prime \prime}\end{array}\right.$ \\
\hline 4 & $7,643.87$ & $13,078.78$ & $3 d_{4}-5 U$ \\
\hline 15 & $7,642.02$ & $13,081.95$ & $\begin{array}{l}1 s_{3}-2 p_{2} \\
3 d-5 V\end{array}$ \\
\hline $\begin{array}{l}1- \\
8 \\
2\end{array}$ & $\begin{array}{l}7,587.6 \\
7,584.66 \\
7,559.78\end{array}$ & $\begin{array}{l}13,175.78 \\
13,180.88 \\
13,224.26\end{array}$ & $\begin{array}{l}3 d_{4}^{\prime}-5 Z \\
3 d_{4}^{\prime}-5 W\end{array}$ \\
\hline $\begin{array}{l}2 \\
2 \\
2 \\
3 \\
1\end{array}$ & $\begin{array}{l}7,501.10 \\
7,492.17 \\
7,474.04 \\
7,471.94 \\
7,451.02\end{array}$ & $\begin{array}{l}13,227.71 \\
13,343.60 \\
13,375.97 \\
13,379.72 \\
13,417.29\end{array}$ & $\begin{array}{l}2 p_{9}-s_{1}^{\prime \prime} \\
2 p_{8}-s_{1}^{\prime \prime \prime \prime} \\
3 d_{5}-5 X \\
3 d_{5}-5 Y \\
3 d_{5}-5 V\end{array}$ \\
\hline $\begin{array}{l}1 \\
2 \\
3 \\
6 \\
5\end{array}$ & $\begin{array}{l}7,441.84 \\
7,424.0 \\
7,400.38 \\
7,393.77 \\
7,385.98\end{array}$ & $\begin{array}{l}13,433.84 \\
13,466.12 \\
13,509.10 \\
13,521.18 \\
13,535.44\end{array}$ & $\begin{array}{l}2 p_{6}-5 d_{4} \\
2 p_{6}-5 d_{2} \\
2 p_{6}-5 d_{1}^{\prime \prime} \\
2 p_{6}-5 d_{1}^{\prime \prime} \\
2 p_{10}-3 s_{5}\end{array}$ \\
\hline $\begin{array}{l}2 \\
4 \\
5 \\
5\end{array}$ & $\begin{array}{l}7,355.54 \\
7,336.44 \\
7,321.46 \\
7,316.28\end{array}$ & $\begin{array}{l}13,591.45 \\
13,626.85 \\
13,654.73 \\
13,664.39\end{array}$ & $\begin{array}{l}3 d_{6}-5 X \\
2 p_{9}-s_{1}^{\prime \prime} \\
1 s_{2}-4 X \\
1 s_{2}-4 Y\end{array}$ \\
\hline
\end{tabular}


TABLE 14.-List of $X e_{\mathrm{x}}$ lines-Continued

\begin{tabular}{|c|c|c|c|}
\hline Intensity & Wave length & Wave number & Combination \\
\hline $\begin{array}{l}5 \\
4 \\
2 \\
1 \\
3\end{array}$ & $\begin{array}{l}7,285.29 \\
7,283.95 \\
7,266.50 \\
7,262.5 \\
7,257.94\end{array}$ & $\begin{array}{l}13,722.51 \\
13,725.04 \\
13,758.00 \\
13,765.57 \\
13,774.22\end{array}$ & $\begin{array}{c}2 p_{7}-4 d_{2} \\
1 s_{2}-4 V \\
2 p_{7}-5 d_{5} \\
2 p_{7}-5 d_{1}^{\prime \prime} \\
3 d_{1}^{\prime \prime}-6 Z\end{array}$ \\
\hline $1^{\circ}$ & $7,244.8$ & $13,799.21$ & \multirow{3}{*}{$\begin{array}{c}3 d_{1}{ }^{\prime \prime}-6 W \\
2 p_{7}-2 s_{3} \\
2 p_{8}-5 d_{4}^{\prime} \\
2 p_{6}-4 s_{5} \\
2 p_{6}-4 s_{4} \\
2 p_{8}-5 d_{4}\end{array}$} \\
\hline 30 & $7,119.58$ & $14,041.91$ & \\
\hline $\begin{array}{l}2 \\
1 \\
3\end{array}$ & $\begin{array}{l}7,047.34 \\
7,035.53 \\
7,018.96\end{array}$ & $\begin{array}{l}14,185.84 \\
14,209.65 \\
14,243.20\end{array}$ & \\
\hline $\begin{array}{r}3 \\
10 \\
2 \\
5 \\
3\end{array}$ & $\begin{array}{l}6,982.04 \\
6,976.17 \\
6,935.59 \\
6,925.52 \\
6,910.80\end{array}$ & $\begin{array}{l}14,318.52 \\
14,330.57 \\
14,414.42 \\
14,435.37 \\
14,466.12\end{array}$ & $\begin{array}{c}2 p_{8}-5 d_{1}{ }^{\prime \prime} \\
2 p_{8}-5 d_{1}{ }^{\prime} \\
3 d_{4}-6 Y \\
3 d_{4}-6 U \\
2 p_{7}-4 s_{4}\end{array}$ \\
\hline $\begin{array}{r}60 \\
20 \\
7 \\
1 \\
3\end{array}$ & $\begin{array}{l}6,882.16 \\
6,872.10 \\
6,866.81 \\
6,863.21 \\
6,860.16\end{array}$ & $\begin{array}{l}14,526.32 \\
14,547.59 \\
14,558.79 \\
14,566.43 \\
14,572.91\end{array}$ & $\begin{array}{c}2 p_{9}-5 d_{4} \\
3 d_{4}{ }^{\prime}-6 Z \\
2 p_{9}-5 d_{2} \\
3 d_{4}{ }^{\prime}-6 \mathrm{~W}\end{array}$ \\
\hline $\begin{array}{r}3 \\
3 \\
10\end{array}$ & $\begin{array}{l}6,850.07 \\
6,848.73 \\
6,846.57\end{array}$ & $\begin{array}{l}14,594.37 \\
14,597.23 \\
14,601.83\end{array}$ & \multirow{3}{*}{$\begin{array}{c}2 p_{9}-5 d_{5} \\
3 d_{1}^{\prime \prime}-7 Z \\
2 p_{9}-5 d_{1}^{\prime \prime} \\
3 d_{1}^{\prime \prime}-7 W \\
2 p_{9}-5 d_{1}^{\prime} \\
1 s_{3}-4 X\end{array}$} \\
\hline 1 & $6,841.30$ & $14,613.08$ & \\
\hline 40 & $6,827.30$ & $14,643.04$ & \\
\hline $\begin{array}{r}1 \\
3 \\
4 \\
2 \\
50\end{array}$ & $\begin{array}{l}6,818.30 \\
6,778.59 \\
6,777.53 \\
6,767.06 \\
6,728.00\end{array}$ & $\begin{array}{l}14,662.37 \\
14,748.27 \\
14,750.56 \\
14,773.40 \\
14,859.16\end{array}$ & $\begin{array}{l}3 d_{5}-6 X \\
3 d_{5}-6 Y \\
3 d_{5}-6 V\end{array}$ \\
\hline $\begin{array}{r}3 \\
4 \\
40 \\
15 \\
3\end{array}$ & $\begin{array}{l}6,681.02 \\
6,678.94 \\
6,668.93 \\
6,666.95 \\
6,657.90\end{array}$ & $\begin{array}{l}14,963.65 \\
14,968.31 \\
14,990.78 \\
14,995.23 \\
15,015.61\end{array}$ & $\begin{array}{c}3 d_{6}-6 Z \\
1 s_{2}-4 p_{10} \\
2 p_{10}-5 d_{6} \\
2 p_{8}-4 s_{5} \\
2 p_{6}-6 d_{2}\end{array}$ \\
\hline $\begin{array}{r}10 \\
1 \\
2 \\
1 \\
20\end{array}$ & $\begin{array}{l}6,632.43 \\
6,608.8 \\
6,607.38 \\
6,602.8 \\
6,595.54\end{array}$ & $\begin{array}{l}15,073.27 \\
15,127.17 \\
15,130.42 \\
15,140.92 \\
15,157.58\end{array}$ & $\begin{array}{c}2 p_{6}-6 d_{5} \\
2 p_{6}-6 d_{1}^{\prime \prime} \\
3 d_{1}^{\prime \prime}-8 Z \\
3 d_{1}^{\prime \prime}-8 W \\
2 p_{6}-6 d_{1}^{\prime}\end{array}$ \\
\hline $\begin{array}{l}1 \\
3 \\
2 \\
5 \\
2\end{array}$ & $\begin{array}{l}6,590.76 \\
6,583.15 \\
6,559.98 \\
6,554.17 \\
6,546.06\end{array}$ & $\begin{array}{l}15,168.56 \\
15,186.11 \\
15,239.75 \\
15,253.26 \\
15,272.16\end{array}$ & $\begin{array}{c}2 p_{7}-6 d_{6} \\
1 s_{2}-4 p_{6} \\
3 d_{4}-7 Y \\
3 d_{4}-7 U \\
2 p_{7}-6 d_{2}\end{array}$ \\
\hline $\begin{array}{r}5 \\
10 \\
5\end{array}$ & $\begin{array}{l}6,543.32 \\
6,533.15 \\
6,521.50\end{array}$ & $\begin{array}{l}15,278.55 \\
15,302.33 \\
15,329.67\end{array}$ & \multirow{3}{*}{$\begin{array}{c}2 p_{9}-4 s_{5} \\
2 p_{9}-4 s_{4} \\
2 p_{7}-6 d_{5} \\
3 d_{4}-7 Z \\
1 s_{2}-4 p_{5}\end{array}$} \\
\hline $15 d$ & $6,504.16$ & $15,370.54$ & \\
\hline $1-$ & $6,500.23$ & $15,379.83$ & \\
\hline
\end{tabular}


TABLE 14.-List of $X e_{\mathrm{I}}$ lines-Continued

\begin{tabular}{|c|c|c|c|}
\hline Intensity & Wave length & Wave number & Combination \\
\hline $\begin{array}{r}15 \\
1 \\
20 \\
20 \\
40\end{array}$ & $\begin{array}{l}6,498.71 \\
6,497.3 \\
6,487.75 \\
6,472.82 \\
6,469.71\end{array}$ & $\begin{array}{l}15,383.43 \\
15,386.76 \\
15,409.41 \\
15,444.96 \\
15,452.38\end{array}$ & $\begin{array}{l}2 p_{7}-6 d_{1}{ }^{\prime \prime} \\
3 d_{4}-7 \cdot W \\
2 p_{10}-5 d_{2} \\
2 p_{10}-5 d_{5} \\
2 p_{10}-5 d_{1}^{\prime \prime}\end{array}$ \\
\hline $\begin{array}{l}1 \\
1- \\
2 \\
3\end{array}$ & $\begin{array}{l}6,451.6 \\
6,450.10 \\
6,430.05 \\
6,418.93\end{array}$ & $\begin{array}{l}15,495.76 \\
15,499.36 \\
15,547.69 \\
15,574.62\end{array}$ & $\begin{array}{r}3 d_{1}{ }^{\prime \prime}-9 Z \\
2 p_{6}-5 s_{5} \\
3 d_{5}-7 X\end{array}$ \\
\hline 2 & $6,418.47$ & $15,575.74$ & $\left\{\begin{array}{l}3 d_{5}-7 Y \\
2 p_{6}-5 s_{4}\end{array}\right.$ \\
\hline $\begin{array}{l}1 \\
2 \\
1- \\
1 \\
2\end{array}$ & $\begin{array}{l}6,412.3 \\
6,355.72 \\
6,344.79 \\
6,337.5 \\
6,333.88\end{array}$ & $\begin{array}{l}15,590.73 \\
15,729.52 \\
15,756.61 \\
15,774.74 \\
15,783.76\end{array}$ & $\begin{array}{c}3 d_{5}-7 V \\
2 p_{10}-2 s_{3} \\
3 d_{1}^{\prime \prime}-10 Z \\
3 d_{4}-8 Y \\
3 d_{4}-8 U\end{array}$ \\
\hline $\begin{array}{r}2 \\
30 \\
1 \\
1 \\
5\end{array}$ & $\begin{array}{l}6,331.37 \\
6,318.08 \\
6,314.88 \\
6,294.36 \\
6,292.62\end{array}$ & $\begin{array}{l}15,790.01 \\
15,823.23 \\
15,831.24 \\
15,882.85 \\
15,887.25\end{array}$ & $\begin{array}{l}3 d_{6}-7 Z \\
2 p_{8}-6 d_{4}{ }^{\prime} \\
2 p_{7}-5 s_{4} \\
2 p_{9}-6 d_{4}\end{array}$ \\
\hline $\begin{array}{l}3 \\
1 \\
1 \\
1 \\
4\end{array}$ & $\begin{array}{l}6,286.02 \\
6,281.7 \\
6,277.2 \\
6,273.15 \\
6,265.28\end{array}$ & $\begin{array}{l}15,903.93 \\
15,914.86 \\
15,926.27 \\
15,936.56 \\
15,956.57\end{array}$ & $\begin{array}{l}3 d_{4}{ }^{\prime}-8 Z \\
3 d_{4}^{\prime}-8 W \\
2 p_{8}-6 d_{1}{ }^{\prime \prime} \\
1 s_{3}-4 p_{10}\end{array}$ \\
\hline $\begin{array}{l}5 \\
1 \\
3\end{array}$ & $\begin{array}{l}6,261.18 \\
6,241.98 \\
6,224.11\end{array}$ & $\begin{array}{l}15,967.02 \\
16,016.14 \\
16,062.12\end{array}$ & $\begin{array}{l}2 p_{8}-6 d_{1}{ }^{\prime} \\
2 p_{6}-7 d_{2} \\
2 p_{6}-7 d_{5}\end{array}$ \\
\hline 2 & $6,206.21$ & $16,108.45$ & $\begin{array}{l}3 d_{5}-8 X \\
2 p_{9}-6 d_{2}\end{array}$ \\
\hline 4 & $6,200.86$ & $16,122.34$ & $2 p_{6}-7 d_{1}^{\prime}$ \\
\hline $\begin{array}{l}8 \\
1 ? \\
1 \\
20 \\
7\end{array}$ & $\begin{array}{l}6,198.25 \\
6,191.3 \\
6,189.06 \\
6,182.41 \\
6,179.66\end{array}$ & $\begin{array}{l}16,129.13 \\
16,147.24 \\
16,153.08 \\
16,170.46 \\
16,177.66\end{array}$ & $\begin{array}{c}2 p_{10}-4 s_{5} \\
3 d_{4}-9 U \\
2 p_{10}-4 s_{4} \\
2 p_{9}-6 d_{4} \\
1 s_{2}-5 X\end{array}$ \\
\hline 9 & $6,178.30$ & $16,181.21$ & $1 s_{2}-5 Y$ \\
\hline $15 d$ & $6,163.78$ & $16,219.33$ & $\left\{\begin{array}{c}1 s_{2}-5 V \\
2 p_{9}-6 d_{1}^{\prime \prime}\end{array}\right.$ \\
\hline $\begin{array}{l}2 \\
1 \\
1\end{array}$ & $\begin{array}{l}6,152.03 \\
6,144.91 \\
6,126.32\end{array}$ & $\begin{array}{l}16,250.31 \\
16,269.14 \\
16,318.51\end{array}$ & $\begin{array}{c}2 p_{9}-6 d_{1}{ }^{\prime} \\
3 d^{\prime}{ }_{4}-9 Z \\
2 p_{7}-7 d_{5}\end{array}$ \\
\hline $\begin{array}{l}1- \\
1\end{array}$ & $\begin{array}{l}6,123.64 \\
6,114.7\end{array}$ & $\begin{array}{l}16,325.65 \\
16,349.52\end{array}$ & $3 d_{6}-8 X$ \\
\hline 5 & $6,111.82$ & $16,357.22$ & $\begin{array}{l}2 p_{8}-5 s_{5} \\
2 p_{7}-7 d_{1}^{\prime \prime}\end{array}$ \\
\hline $\begin{array}{l}1- \\
1\end{array}$ & $\begin{array}{l}6,108.52 \\
6,047.9\end{array}$ & $\begin{array}{l}16,366.06 \\
16,530.10\end{array}$ & $\begin{array}{l}2 p_{0}-6 s_{5} \\
3 d_{4}-10 Z\end{array}$ \\
\hline $\begin{array}{l}1 \\
1- \\
2 \\
3 \\
1\end{array}$ & $\begin{array}{l}6,043.2 \\
6,009.41 \\
6,007.80 \\
5,998.07 \\
5,989.11\end{array}$ & $\begin{array}{l}16,542.95 \\
16,635.96 \\
16,640.43 \\
16,667.42 \\
16,692.36\end{array}$ & $\begin{array}{l}2 p_{7}-6 s_{4} \\
2 p_{9}-5 s_{5} \\
2 p_{9}-5 s_{4} \\
2 p_{6}-8 d_{5}\end{array}$ \\
\hline
\end{tabular}


TABLE 14.-List of $X e_{\mathbf{r}}$ lines-Continued

\begin{tabular}{|c|c|c|c|}
\hline Intensity & Wave length & Wave number & Combination \\
\hline 2 & $5,974.09$ & $16,734.33$ & $\left\{\begin{array}{l}2 d_{5}-10 X Y \\
2 p_{-}-8 d^{\prime}\end{array}\right.$ \\
\hline $\begin{array}{c}10 \\
8 \\
1- \\
2\end{array}$ & $\begin{array}{l}5,934.15 \\
5,931.23 \\
5,925.33 \\
5,922.51\end{array}$ & $\begin{array}{l}16,846.96 \\
16,855.25 \\
16,872.03 \\
16,880.07\end{array}$ & $\begin{array}{c}2 p_{8}-7 d_{4}^{\prime} \\
2 p_{10}-6 d_{6} \\
2 p_{9}-7 d_{4}\end{array}$ \\
\hline 1 & $5,921.79$ & $16,882.12$ & $1 s_{2}-5 p_{10}$ \\
\hline 2 & $5,904.42$ & 16, 931. 78 & $\begin{array}{l}2 d_{3}-11 X Y \\
2 p_{8}-7 d_{1}^{\prime}\end{array}$ \\
\hline $\begin{array}{r}15 \\
1 \\
15\end{array}$ & $\begin{array}{l}5,894.99 \\
5,889.11 \\
5,875.02\end{array}$ & $\begin{array}{l}16,958.87 \\
16,975.80 \\
17,016.51\end{array}$ & $\begin{array}{l}2 p_{10}-6 d_{2} \\
2 p_{7}-8 d_{1}^{\prime \prime} \\
2 p_{10}-6 d_{5}\end{array}$ \\
\hline $\begin{array}{l}2 \\
1- \\
1 \\
1 \\
1\end{array}$ & $\begin{array}{l}5,856.43 \\
5,849.47 \\
5,843.4 \\
5,840.6 \\
5,830.48\end{array}$ & $\begin{array}{l}17,070.53 \\
17,090.83 \\
17,108.59 \\
17,116.80 \\
17,146.50\end{array}$ & $\begin{array}{c}2 p_{10}-6 d_{1}{ }^{\prime \prime} \\
2 p_{9}-7 d_{2} \\
2 p_{6}-9 d_{5} \\
2 p_{6}-9 d_{1}{ }^{\prime}\end{array}$ \\
\hline $\begin{array}{r}6 \\
20 \\
1 \\
3 \\
1\end{array}$ & $\begin{array}{l}5,824.78 \\
5,823.89 \\
5,820.52 \\
5,814.49 \\
5,807.2\end{array}$ & $\begin{array}{l}17,163.28 \\
17,165.91 \\
17,175.84 \\
17,193.66 \\
17,215.24\end{array}$ & $\begin{array}{l}2 p_{9}-7 d_{4} \\
1 s_{3}-5 X \\
2 p_{8}-6 s_{5} \\
2 p_{9}-7 d_{1}{ }^{\prime \prime} \\
2 p_{9}-7 d_{1}^{\prime}\end{array}$ \\
\hline $\begin{array}{l}1 \\
1 \\
1 \\
1\end{array}-$ & $\begin{array}{l}5,748.1 \\
5,740.2 \\
5,733.4 \\
5,726.0\end{array}$ & $\begin{array}{l}17,392.24 \\
17,416.72 \\
17,436.83 \\
17,459.37\end{array}$ & $\begin{array}{l}2 p_{7}-9 d_{1}^{\prime \prime} \\
2 p_{6}-10 d_{5} \\
2 p_{6}-10 d_{1}^{\prime} \\
2 p_{9}-6 s_{5}\end{array}$ \\
\hline 1 & $5,722.1$ & $17,471.27$ & $\begin{array}{l}2 p_{6}-10 d_{5} \\
2 p_{9}-6 s_{4}\end{array}$ \\
\hline $\begin{array}{l}4 \\
2 \\
1 \\
1- \\
1-\end{array}$ & $\begin{array}{l}5,716.14 \\
5,715.71 \\
5,709.6 \\
5,706.8 \\
5,698.4\end{array}$ & $\begin{array}{l}17,489.48 \\
17,490.80 \\
17,509.52 \\
17,518.11 \\
17,543.93\end{array}$ & $\begin{array}{c}2 p_{8}-8 d_{4}^{\prime} \\
2 p_{10}-5 s_{5} \\
2 p_{9}-8 d_{4} \\
2 p_{10}-5 s_{4} \\
2 p_{8}-8 d_{1}^{\prime}\end{array}$ \\
\hline $\begin{array}{l}3 \\
5 \\
2 \\
3 \\
1\end{array}$ & $\begin{array}{l}5,696.44 \\
5,695.74 \\
5,688.31 \\
5,618.80 \\
5,612.5\end{array}$ & $\begin{array}{l}17,549.96 \\
17,552.12 \\
17,575.05 \\
17,792.47 \\
17,812.44\end{array}$ & $\begin{array}{l}1 s_{2}-6 X \\
1 s_{2}-6 Y \\
1 s_{2}-6 V \\
2 p_{9}-8 d_{4} \\
2 p_{9}-8 d_{1}^{\prime \prime}\end{array}$ \\
\hline $\begin{array}{l}1 \\
3 \\
2 \\
4 \\
4\end{array}$ & $\begin{array}{l}\text { 5, 594. } 21 \\
5,581.70 \\
5,579.19 \\
5,566.60 \\
5,552.33\end{array}$ & $\begin{array}{l}17,870.68 \\
17,910.73 \\
17,918.78 \\
17,959.31 \\
18,005.47\end{array}$ & $\begin{array}{c}1 s_{3}-5 p_{10} \\
2 p_{10}-7 d_{6} \\
2 p_{8}-9 d_{4}^{\prime} \\
2 p_{10}-7 d_{2} \\
2 p_{7}-7 d_{5}\end{array}$ \\
\hline $1-$ & $5,540.2$ & $18,044.89$ & $\left\{\begin{array}{c}1 s_{2}-6 p_{6} \\
2 p_{10}-7 d_{1}^{\prime \prime}\end{array}\right.$ \\
\hline $\begin{array}{l}2 \\
1 \\
1 \\
1\end{array}$ & $\begin{array}{l}5,488.53 \\
5,487.05 \\
5,484.2 \\
5,440.2\end{array}$ & $\begin{array}{l}18,214.77 \\
18,219.68 \\
18,229.15 \\
18,376.58\end{array}$ & $\begin{array}{l}2 p_{9}-9 d_{4} \\
2 p_{8}-10 d_{4}^{\prime}{ }^{\prime} \\
2 p_{9}-9 d_{1}^{\prime \prime} \\
1 s_{2}-7 X\end{array}$ \\
\hline $\begin{array}{l}1 \\
1 \\
1 \\
1 \\
2\end{array}$ & $\begin{array}{l}5,439.9 \\
5,435.6 \\
5,418.0 \\
5,400.4 \\
5,394.68\end{array}$ & $\begin{array}{l}18,377.60 \\
18,392.13 \\
18,451.88 \\
18,512.01 \\
18,531.64\end{array}$ & $\begin{array}{c}1 s_{2}-7 Y \\
1 s_{2}-7 V \\
\\
2 p_{9}-10 d_{4} \\
2 p_{10}-8 d_{6}\end{array}$ \\
\hline
\end{tabular}


TABLE 14.-List of $X e_{1}$ lines-Continued

\begin{tabular}{|c|c|c|c|}
\hline Intensity & Wave length & Wave number & Combination \\
\hline $\begin{array}{l}4 \\
1- \\
1 \\
2 \\
2\end{array}$ & $\begin{array}{l}\text { 5, 392. } 74 \\
5,368.99 \\
5,367.0 \\
5,364.55 \\
5,362.13\end{array}$ & $\begin{array}{l}18,538.31 \\
18,620.31 \\
18,627.22 \\
18,635.72 \\
18,644.14\end{array}$ & $\begin{array}{l}2 p_{10-8 d_{5}} \\
2 p_{10}-8 d_{2}\end{array}$ \\
\hline $\begin{array}{l}1- \\
1- \\
1 \\
1 \\
1\end{array}$ & $\begin{array}{l}5,328.5 \\
5,306.23 \\
5,286.1 \\
5,249.0 \\
5,245.2\end{array}$ & $\begin{array}{l}18,761.80 \\
18,840.55 \\
18,912.29 \\
19,045.96 \\
19,059.76\end{array}$ & $\begin{array}{r}1 s_{2}-8 Y \\
2 p_{10}-9 d_{2} \\
2 p_{10}-9 d_{5}\end{array}$ \\
\hline $\begin{array}{r}1- \\
2 \\
15 \\
1 \\
60\end{array}$ & $\begin{array}{l}5,185.33 \\
5,162.66 \\
5,028.26 \\
5,023.84 \\
4,923.15\end{array}$ & $\begin{array}{l}19,279.82 \\
19,364.48 \\
19,882.07 \\
19,899.56 \\
20,306.55\end{array}$ & $\begin{array}{l}1 s_{2}-9 Y \\
1 s_{3}-7 X \\
1 s_{4}-3 p_{10} \\
1 s_{3}-8 X Y \\
1 s_{4}-3 p_{9}\end{array}$ \\
\hline $\begin{array}{r}50 \\
60 \\
70 \\
100 \\
20\end{array}$ & $\begin{array}{l}4,916.50 \\
4,843.29 \\
4,829.71 \\
4,807.01 \\
4,792.61\end{array}$ & $\begin{array}{l}20,334.01 \\
20,641.37 \\
20,669.41 \\
20,797.16 \\
20,859.64\end{array}$ & $\begin{array}{l}1 s_{4}-2 p_{4} \\
1 s_{4}-3 p_{6} \\
1 s_{4}-3 p_{7} \\
1 s_{4}-3 p_{5} \\
1 s_{5}-3 p_{10}\end{array}$ \\
\hline $\begin{array}{c}1- \\
1 h ? \\
100 \\
2 \\
2\end{array}$ & $\begin{array}{l}4,755.69 \\
4,746.40 \\
4,734.15 \\
4,721.01 \\
4,708.19\end{array}$ & $\begin{array}{l}21,017.17 \\
21,062.73 \\
21,117.23 \\
21,176.00 \\
21,233.66\end{array}$ & $\begin{array}{l}1 s_{4}-2 p_{3} \\
1 s_{4}-2 p_{2}\end{array}$ \\
\hline $\begin{array}{l}70 \\
25\end{array}$ & $\begin{array}{l}4,697.02 \\
4,690.97\end{array}$ & $\begin{array}{l}21,284.16 \\
21,311.61\end{array}$ & $\begin{array}{l}1 s_{5}-3 p_{9} \\
1 s_{5}-2 p_{4}\end{array}$ \\
\hline $\begin{array}{r}300 \\
1 \\
200\end{array}$ & $\begin{array}{l}4,671.22 \\
4,636.55 \\
4,624.29\end{array}$ & $\begin{array}{l}21,401.71 \\
21,561.74 \\
21,618.90\end{array}$ & $\begin{array}{l}1 s_{5}-3 p_{8} \\
1 s_{5}-3 p_{6}\end{array}$ \\
\hline $\begin{array}{r}1 \\
8 \\
30 \\
1 \\
50\end{array}$ & $\begin{array}{l}4,621.36 \\
4,611.88 \\
4,582.74 \\
4,574.86 \\
4,524.68\end{array}$ & $\begin{array}{l}21,632.61 \\
21,677.08 \\
21,814.92 \\
21,852.49 \\
22,094.83\end{array}$ & $\begin{array}{l}1 s_{5}-3 p_{7} \\
1 s_{4}-2 p_{1} \\
1 s_{5}-2 p_{3}\end{array}$ \\
\hline $\begin{array}{r}100 \\
10 \\
15 \\
3 \\
1\end{array}$ & $\begin{array}{l}4,500.99 \\
4,385.75 \\
4,383.90 \\
4,372.25 \\
4,215.5\end{array}$ & $\begin{array}{r}22,211.12 \\
22,794.73 \\
22,804.35 \\
\therefore \quad 22,865.11 \\
23,715.32\end{array}$ & $\begin{array}{l}1 s_{5}-2 p_{2} \\
1 s_{4}-4 X \\
1 s_{4}-4 Y \\
1 s_{4}-4 V\end{array}$ \\
\hline $\begin{array}{r}3 \\
10 \\
30 \\
1 \\
4\end{array}$ & $\begin{array}{l}4,205.37 \\
4,203.69 \\
4,193.51 \\
4,192.99 \\
4,135.12\end{array}$ & $\begin{array}{l}23,772.45 \\
23,781.95 \\
23,839.68 \\
23,842.62 \\
24,176.30\end{array}$ & $\begin{array}{l}1 s_{5}-4 X \\
1 s_{5}-4 Y \\
1 s_{5}-4 U \\
1 s_{5}-4 V \\
1 s_{4}-4 p_{9}\end{array}$ \\
\hline $\begin{array}{r}15 \\
10 \\
20 \\
1 \\
5\end{array}$ & $\begin{array}{l}4,116.11 \\
4,109.71 \\
4,078.81 \\
4,057.80 \\
3,985.22\end{array}$ & $\begin{array}{l}24,287.95 \\
24,325.78 \\
24,510.06 \\
24,636.96 \\
25,085.65\end{array}$ & $\begin{array}{l}1 s_{4}-4 p_{7} \\
1 s_{4}-4 p_{6} \\
1 s_{4}-4 p_{5} \\
1 s_{5}-4 p_{10}\end{array}$ \\
\hline
\end{tabular}


TABLE 14.-List of $X e_{\mathrm{I}}$ lines-Continued

\begin{tabular}{|r|c|c|c|}
\hline Intensity & Wave length & Wave number & Combination \\
\hline & $3,974.42$ & $25,153.81$ & $1 s_{5}-4 p_{9}$ \\
30 & $3,967.55$ & $25,197.37$ & $1 s_{5}-4 p_{8}$ \\
1 & $3,956.88$ & $25,265.31$ & $1 s_{5}-4 p_{7}$ \\
20 & $3,950.92$ & $25,303.43$ & $1 s_{5}-4 p_{6}$ \\
1 & $3,948.70$ & $25,317.65$ & $1 s_{4}-6 X$ \\
& & & \\
5 & $3,948.19$ & $25,320.92$ & $1 s_{4}-5 Y$ \\
1 & $3,842.4$ & $26,018.05$ & \\
3 & $3,826.86$ & $26,123.70$ & $1 s_{4}-5 p_{7}$ \\
2 & $3,823.76$ & $26,144.88$ & $1 s_{4}-5 p_{6}$ \\
4 & $3,809.90$ & $26,239.99$ & $1 s_{4}-5 p_{5}$ \\
& & & \\
3 & $3,801.43$ & $26,298.45$ & $1 s_{5}-5 Y$ \\
10 & $3,796.33$ & $26,333.78$ & $1 s_{5}-5 U$ \\
2 & $3,745.44$ & $26,691.57$ & $1 s_{4}-6 Y$ \\
1 & $3,702.72$ & $26,999.52$ & $1 s_{5}-5 p_{10}$ \\
1 & $3,696.82$ & $27,042.61$ & $1 s_{5}-5 p_{9}$ \\
5 & $3,693.50$ & $27,066.92$ & $1 s_{5}-5 p_{8}$ \\
4 & $3,685.91$ & $27,122.65$ & $1 s_{5}-5 p_{6}$ \\
1 & $3,679.31$ & $27,171.30$ & $1 s_{4}-6 p_{7}$ \\
$1-$ & $3,677.62$ & $27,183.79$ & $1 s_{4}-6 p_{6}$ \\
2 & $3,669.94$ & $27,240.67$ & $1 s_{4}-6 p_{5}$ \\
2 & $3,633.15$ & $27,516.51$ & $1 s_{4}-7 Y$ \\
2 & $3,613.07$ & $27,669.43$ & $1 s_{5}-6 Y$ \\
3 & $3,610.32$ & $27,690.51$ & $1 s_{5}-6 U$ \\
2 & $3,554.03$ & $28,129.07$ & $1 s_{5}-6 p_{8}$ \\
2 & $3,549.84$ & $28,162.27$ & $1 s_{5}-6 p_{6}$ \\
1 & $3,506.75$ & $28,508.31$ & $1 s_{5}-7 U$ \\
1 & $3,472.43$ & $28,790.06$ & $1 s_{5}-7 p_{8}$ \\
1 & $3,469.96$ & $28,810.55$ & $1 s_{5}-7 p_{6}$ \\
$1-$ & $3,442.77$ & $29,038.09$ & $1 s_{5}-8 U$ \\
\hline & & & \\
\hline
\end{tabular}

The general features of this classification of $\mathrm{Xe}_{\mathrm{I}}$ lines parallel those of the preceding rare gases, $\mathrm{Ne}_{\mathrm{I}}, \mathrm{A}_{\mathrm{I}}, \mathrm{Kr}_{\mathrm{I}}$, and are also in accord with the structure expected on theoretical grounds. Many resemblances among these four spectra have been alluded to in this paper, but we shall reserve further discussion and interpretation of them so as to include comparisons with the fifth spectrum, that of radon, which is now under investigation.

The cooperation of the authors has been made possible in this investigation through the grant of a research fellowship to one of them (T. L. deB.) by the International Education Board.

Washington, May 10, 1929. 